\title{
Laser Line Scan Characterization of Cavitation-Induced Erosion to SNS Mercury Target Vessels
}

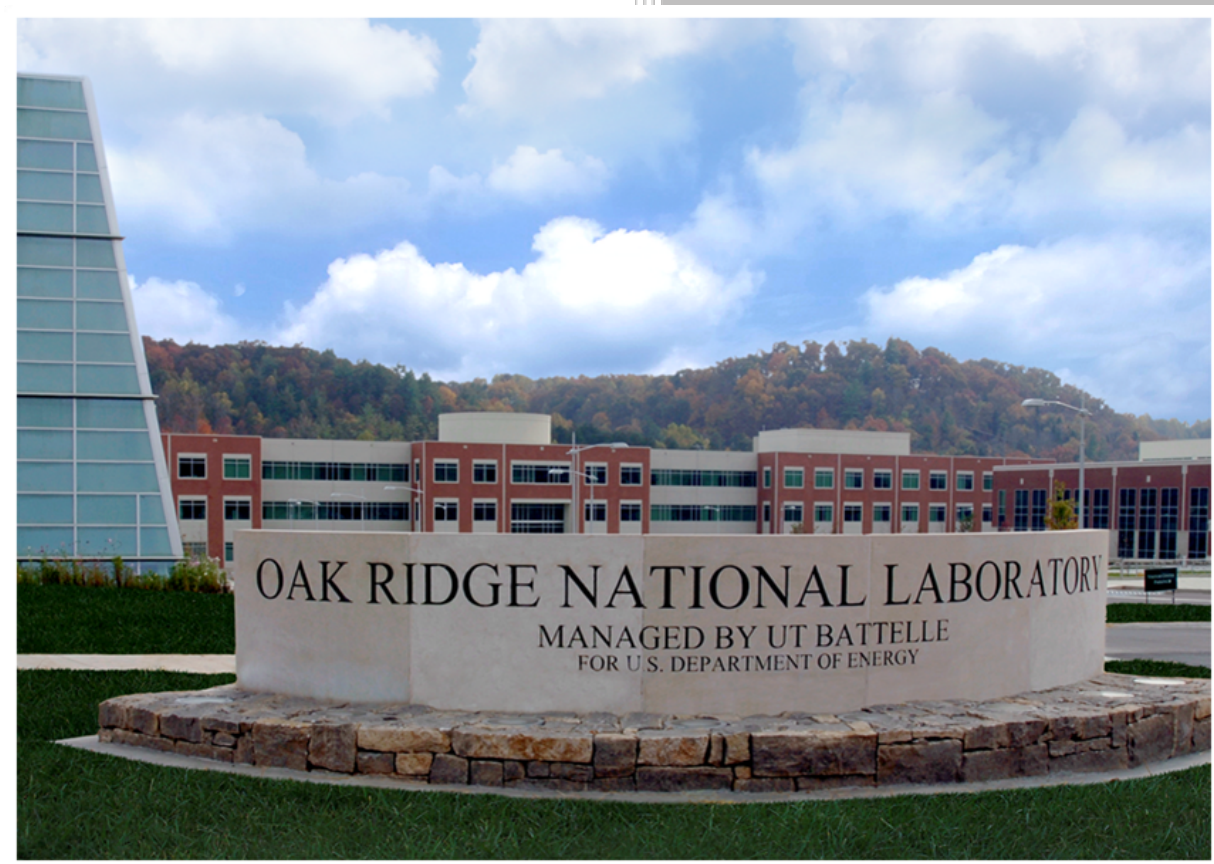

David McClintock

Doug Bruce

Richard Schwartz

Timothy Carroll

Mike Dayton

Drew Winder

January 2019

Approved for public release Distribution is unlimited. 


\title{
DOCUMENT AVAILABILITY
}

Reports produced after January 1, 1996, are generally available free via US Department of Energy (DOE) SciTech Connect.

Website http://www.osti.gov/scitech/

Reports produced before January 1, 1996, may be purchased by members of the public from the following source:

\author{
National Technical Information Service \\ 5285 Port Royal Road \\ Springfield, VA 22161 \\ Telephone 703-605-6000 (1-800-553-6847) \\ TDD 703-487-4639 \\ Fax 703-605-6900 \\ E-mail info@ntis.gov \\ Website http://classic.ntis.gov/
}

Reports are available to DOE employees, DOE contractors, Energy Technology Data Exchange representatives, and International Nuclear Information System representatives from the following source:

Office of Scientific and Technical Information

PO Box 62

Oak Ridge, TN 37831

Telephone 865-576-8401

Fax 865-576-5728

E-mail reports@osti.gov

Website http://www.osti.gov/contact.html

This report was prepared as an account of work sponsored by an agency of the United States Government. Neither the United States Government nor any agency thereof, nor any of their employees, makes any warranty, express or implied, or assumes any legal liability or responsibility for the accuracy, completeness, or usefulness of any information, apparatus, product, or process disclosed, or represents that its use would not infringe privately owned rights. Reference herein to any specific commercial product, process, or service by trade name, trademark, manufacturer, or otherwise, does not necessarily constitute or imply its endorsement, recommendation, or favoring by the United States Government or any agency thereof. The views and opinions of authors expressed herein do not necessarily state or reflect those of the United States Government or any agency thereof. 
Neutron Technologies Division

Spallation Neutron Source

Oak Ridge National Laboratory

\title{
LASER Line SCAN CHARACTERIZATION OF CAVITATION-INDUCED EROSION TO SNS MERCURY TARGET VESSELS
}

\author{
David McClintock \\ Doug Bruce \\ Richard Schwartz \\ Timothy Carroll \\ Mike Dayton \\ Drew Winder
}

Date Published: January 2019

\author{
Prepared by \\ OAK RIDGE NATIONAL LABORATORY \\ Oak Ridge, TN 37831-6283 \\ managed by \\ UT-BATTELLE, LLC \\ for the \\ US DEPARTMENT OF ENERGY \\ under contract DE-AC05-00OR22725
}





\section{CONTENTS}

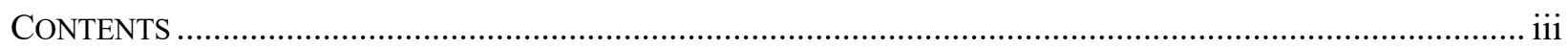

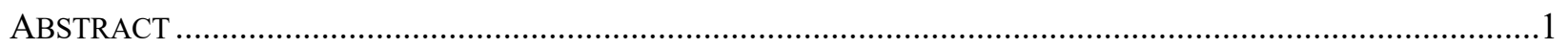

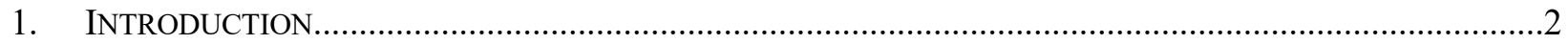

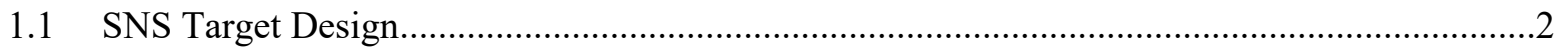

1.2 Cavitation-Induced Erosion Damage in SNS Targets........................................................

1.3 Post Irradiation Examination Observations of SNS Targets...................................................

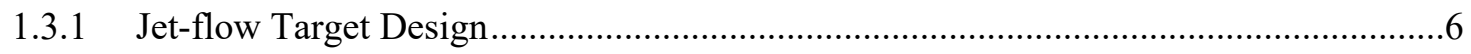

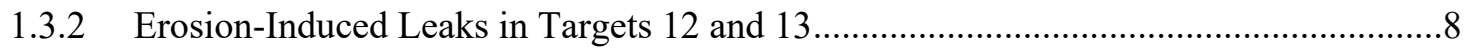

1.3.3 Characterization of Cavitation-Induced Erosion Damage .......................................11

1.3.4 Implementation of the Target Management Plan..................................................... 12

1.3.5 Observations of Damage in High-Use Jet-Flow Targets ...........................................12

1.3.6 Implementation of Small-Bubble Gas Injection ......................................................13

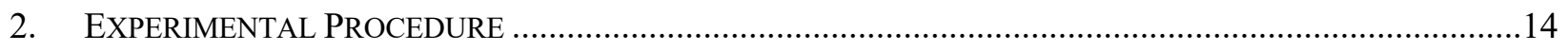

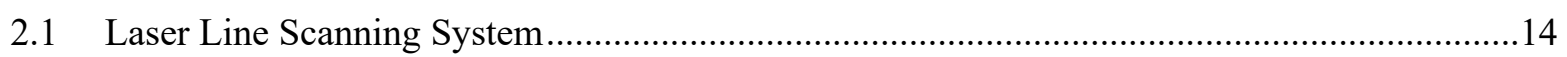

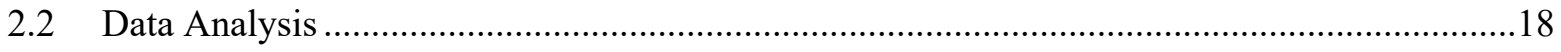

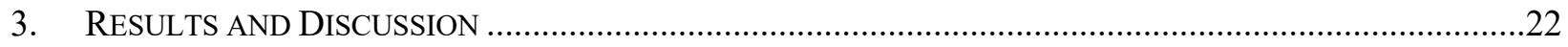

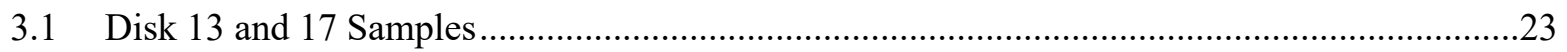

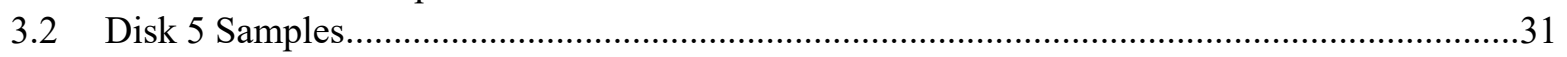

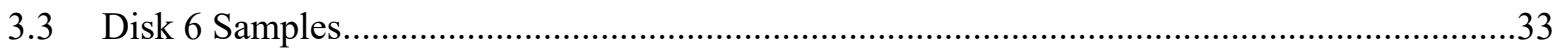

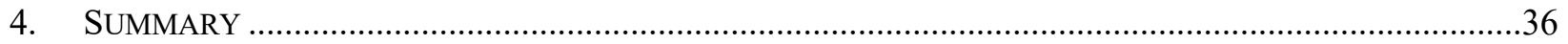

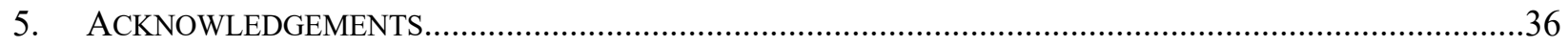

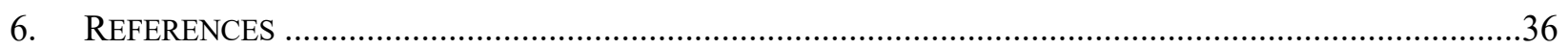




\begin{abstract}
The mercury-facing surfaces of the Spallation Neutron Source (SNS) target vessel are damaged during operation by a phenomenon called cavitation-induced erosion. A post irradiation examination (PIE) program is maintained at the SNS to learn about damage processes that occurred in targets during operation and improve the target design. Samples removed from the beam entrance region of SNS targets were scanned using a laser line scanner to produce point-cloud computer models of the samples, which were analyzed to calculate various erosion metrics and characterize the erosion damage to each sample. The results quantified parameters such as the maximum depth of erosion and the location where the maximum erosion occurred. The scan data also illustrated the differences in erosion patterns between different target designs and operational histories. Results from the scans also showed the maximum depth of erosion was greatly reduced at all locations examined in the SNS targets operated with gas injection. The data produced by these scans will be used to quantify and compare the erosion damage in targets with different design features and operational histories. This report details the experimental procedures, data analysis, and results obtained from scanning 28 SNS target samples.
\end{abstract}




\section{INTRODUCTION}

\subsection{SNS TARGET DESIGN}

Neutrons are produced at the Spallation Neutron Source (SNS) by bombarding liquid mercury flowing through a target vessel with high-energy $(\sim 1 \mathrm{GeV})$ protons at a frequency of $60 \mathrm{~Hz}$. The protons injected into the target module induce spallation reactions in the mercury, which liberate high-energy neutrons from the spalling atoms. The high-energy neutrons produced in the target are moderated to lower energies and guided to neutron-scattering instruments for use in scientific experiments.

Mercury is the spallation target material at the SNS and flows through a $316 \mathrm{~L}$ stainless steel target module, which supplies cool mercury to the target area and returns heated mercury to the heat exchanger process loop. The SNS target module consists of two vessels: a mercury vessel and a water-cooled shroud, as shown in Figure 1. The shroud is designed to catch any mercury that might leak from the mercury vessel during operation. The two vessels are joined at the core vessel seal flange by either welding or a bolted joint. A network of leak detectors is located in the interstitial region between the mercury vessel and the shroud. The leak detectors are designed to activate when in contact with mercury or water and signal to operators that a leak has occurred.

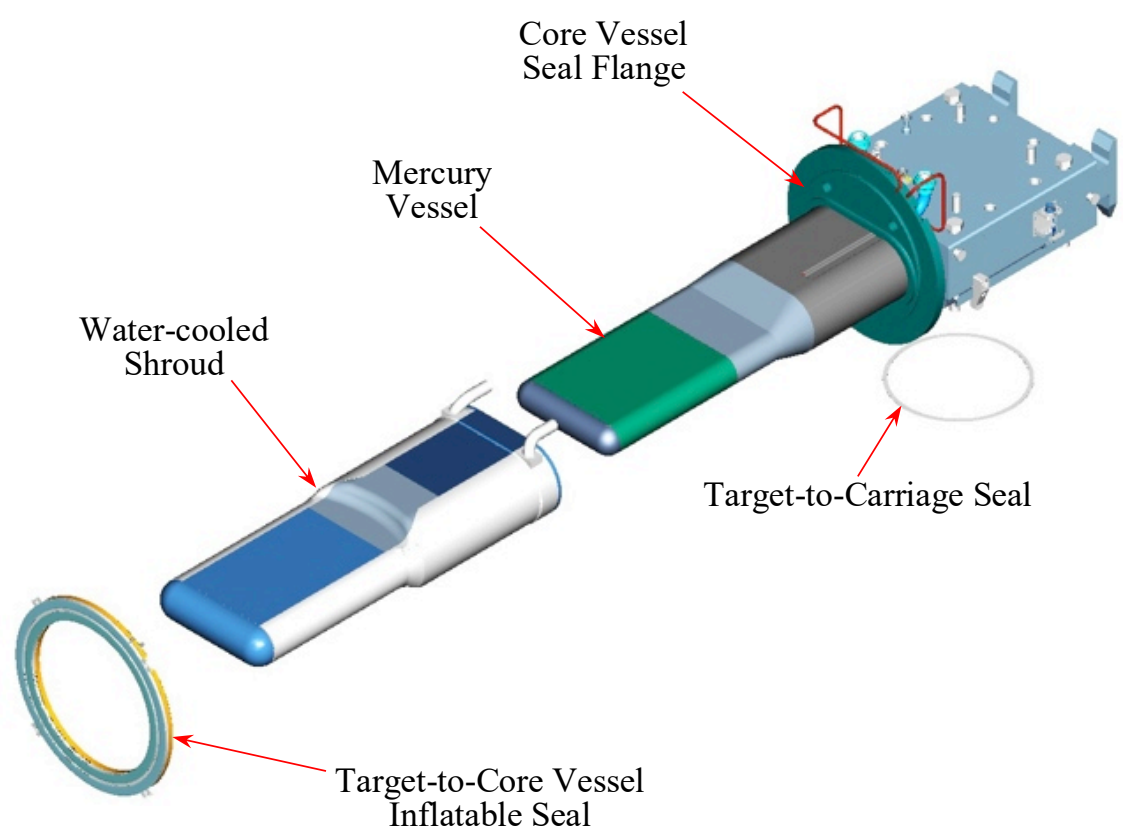

Figure 1. Schematic of water-cooled shroud and mercury vessel of the SNS target module.

Mercury flows through the "original design" SNS target vessel via two pathways: (1) the bulk mercury flow and (2) the mercury window flow. Most the mercury flows through the bulk-flow passages of the SNS target, as shown in Figure 2, and the majority of the neutrons are produced in the bulk flow. The bulk flow enters the target through supply passageways on the sides of the target, interacts with the proton beam at the front of the target, and the heated mercury returns to the mercury process loop through the passageways in the center of the target. 


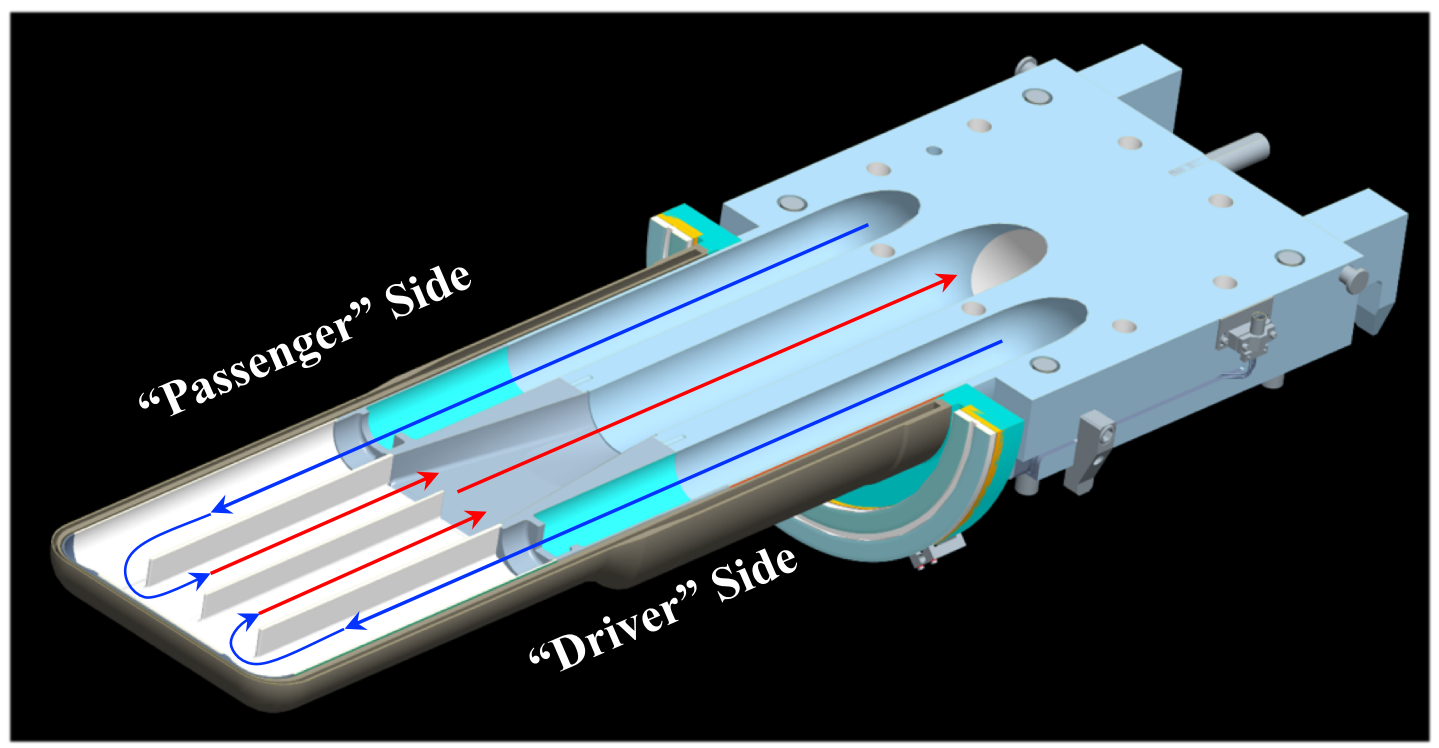

Figure 2. Bulk-flow pathway for mercury through SNS target. Cold mercury enters the target on the side passageways (shown in blue) and the heated mercury exits the target through the center return passageway (shown in red).

The second mercury flow path is the window flow, which enters the target through a 2 in diameter passage on the bottom of the vessel, as shown in Figure 3. The window flow is supplied to the front of the target via narrow passageways that run along the bottom of the target vessel. At the beam entrance region of the target, the window flow passes between the inner and outer walls of the mercury vessel. The window flow was designed to cool the walls of the target vessel beam entrance region during operation and keep the stainless steel temperature below approximately $200^{\circ} \mathrm{C}$ to prevent leaching of alloying elements from the $316 \mathrm{~L}$ target material [1].

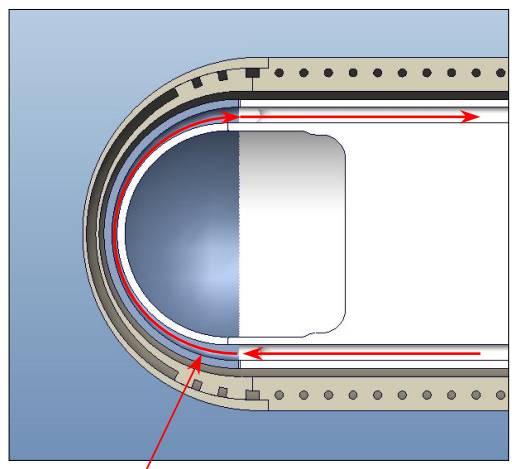

Mercury Window Flow

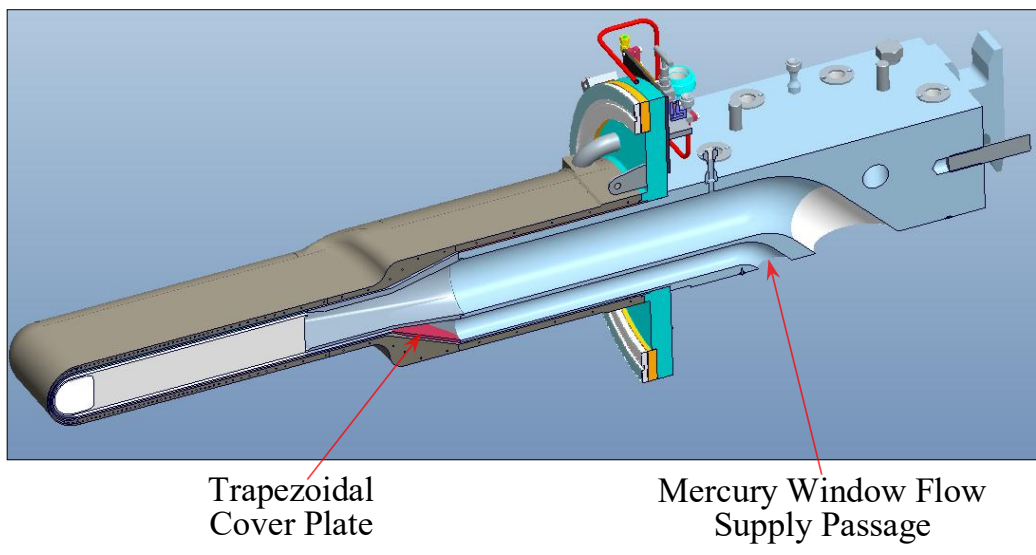

Figure 3. Mercury window-flow pathway in the SNS target. 


\subsection{CAVITATION-INDUCED EROSION DAMAGE IN SNS TARGETS}

The operating environment of the SNS target is quite severe; high-energy particle radiation alters the mechanical properties of the 316L stainless steel and the vessel structure must endure ultra-high cycle fatigue throughout its lifetime. But the SNS target must withstand another severe damage mechanism unique to pulsed liquid metal spallation sources, namely erosion of the vessel surfaces from collapsing cavitation bubbles. The process removes material from the interior surfaces of the target vessel and, to date, has caused leaks to develop in two SNS target vessels: Target 12 and Target 13.

A proton pulse deposits its energy almost instantly into the mercury interaction volume inside the target vessel. The rapid isochoric heating of the mercury causes a compression pressure wave to propagate from the proton interaction volume. The compression waves interact with the interior surfaces of the target vessel to produce tensile pressure waves that propagate throughout the mercury volume. If the tensile pressure waves have sufficient negative pressure, the mercury cavitates and small mercury gas bubbles are nucleated behind the wave. The small bubbles grow until reaching a maximum radius, after which the collapse process begins. As the bubbles collapse a high-velocity jet of mercury may form and pierce through the center of the bubble, as shown in Figure 4.
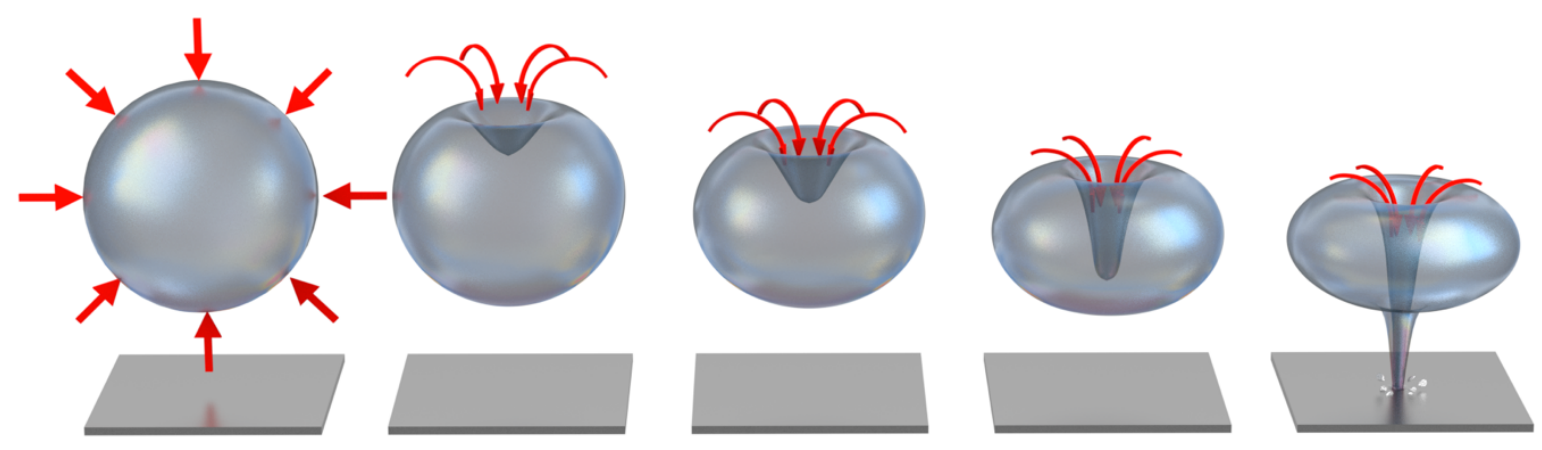

(a)

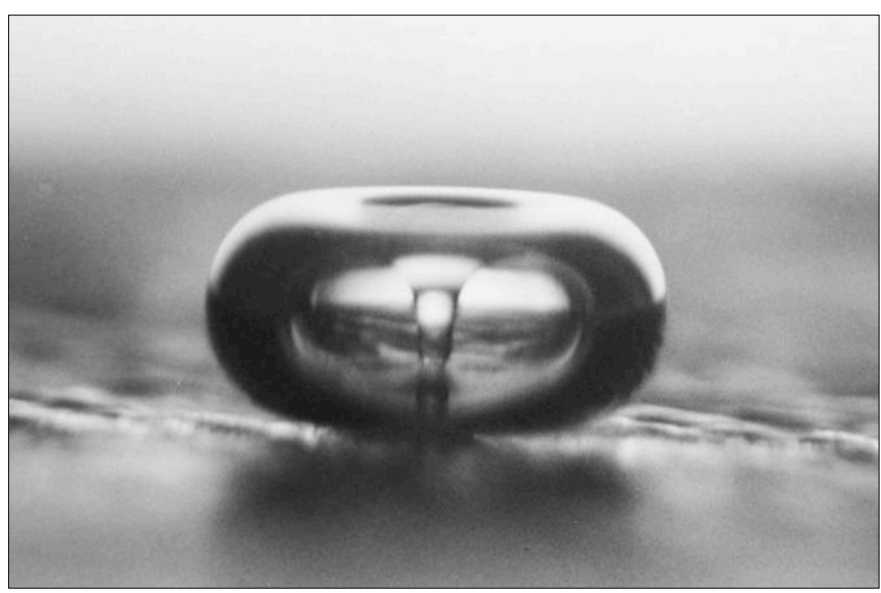

Photograph courtesy of Lawrence Crum (University of Washington)

(b)

Figure 4. (a) Schematic of the cavitation bubble collapse process and (b) photograph of a high-velocity water jet penetrating a collapsing bubble and impinging onto a surface. 
If the bubble collapses near a surface, material can be removed by two processes: (1) direct removal of surface material by the high-velocity jet, and (2) fatigue fracture from repeated shockwave impacts from bubbles that collapse near the surface. This process of material removal by the collapse of cavitation bubbles is called cavitation-induced erosion.

To address cavitation-induced erosion a surface hardening treatment, called Kolsterising ${ }^{\circledR}$, is used to harden the surfaces of the mercury target vessel and inhibit erosion damage. The Kolsterising ${ }^{\circledR}$ treatment is a relatively low-temperature process that produces a supersaturated hardened surface layer, which contains approximately $6 \mathrm{wt} \%$ carbon at the surface which gradually decreases to the base metal carbon concentration ( $\sim 0.02 \mathrm{wt} \% \mathrm{C})$ at a depth of approximately $50 \mu \mathrm{m}$. The uniform steady decrease in carbon concentration produces a layer with a gradual decrease in hardness, and since the structure remains face centered cubic there is no sudden change in microstructure that would promote chipping or removal of the hardened surface layer. The Kolsterising ${ }^{\circledR}$ treatment has been shown to increase the incubation period for cavitation-induced erosion [2], which delays the onset of mass-wastage type of damage and increases the target lifetime. For more detailed descriptions of the Kolsterising ${ }^{\circledR}$ treated layer readers are referred to [25]. To date, all SNS targets operated have been treated with Kolsterising ${ }^{\circledR}$, as will all targets for the foreseeable future.

\subsection{POST IRRADIATION EXAMINATION OBSERVATIONS OF SNS TARGETS}

While laboratory experiments were helpful during early research on cavitation-induced erosion, no facility currently exists that can accurately simulate the operating environment at the SNS, and postoperational targets offer the only opportunity to learn about the degradational processes that occur inside the target during operation. Information on the damage that occurred inside the target during operation is critical to target development efforts. Therefore, post irradiation examination (PIE) characterizations are routinely performed on SNS target vessels after removal from service to study and catalog the erosion damage to the mercury-facing surfaces of the target vessel, to locate leaks that occur during operation, and to measure the change in mechanical properties of the vessel material. PIE activities include removal of disk-shaped samples from the beam entrance region of target vessels [6]; inspection of the target interior via photography and videoprobe examination; photography of the target vessels and samples [7]; and characterization of vessel material tensile properties [8]. Cavitation-induced erosion damage has been observed in SNS targets in the past and readers are referred to [9] for detailed descriptions of cavitationinduced erosion damage observed in SNS target vessels.

PIE observations from early targets showed erosion damage was most severe along the inner wall of the mercury vessel at the beam entrance region of the mercury target vessel. A diagram with the target sample identification scheme and photographs of samples from the inner wall of Target 8 are shown in Figure 5. Target 8 was operated at an average power of approximately $850 \mathrm{~kW}$ to $3750 \mathrm{MW}$-hr and had erosion damage patterns representative of an original design SNS target after service. Erosion damage to the inner wall was concentrated along the vertical centerline where the wall was fractured, and an erosion pattern resembling the Greek letter "phi", $\Phi$, was observed at the center of the beam entrance region. Erosion damage was observed along the window-flow facing surfaces as well, but the pits were generally shallow and did not threaten to penetrate the vessel wall. Regions of appreciable erosion were also observed 
at the extents of the inner wall where the supply passages turn the flow towards the center of the target, but were not considered threatening to the vessel until a leak was discovered in Target 12 at this location.
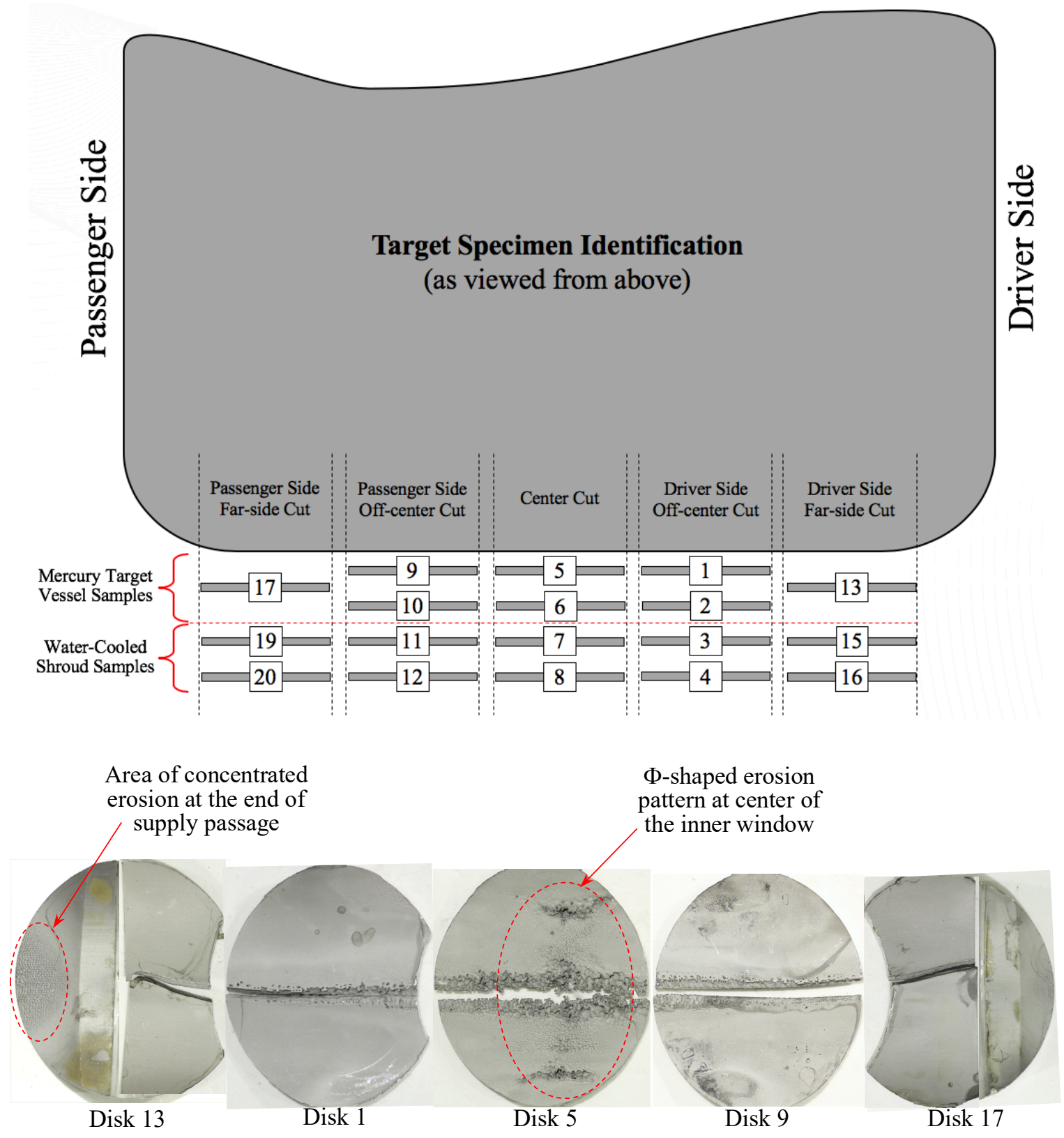

Figure 5. Diagram of target sample identification scheme and photographs of disk-shaped samples from the Target 8 mercury vessel inner wall.

\subsubsection{JET-FLOW TARGET DESIGN}

Another technique implemented in the SNS target to mitigate erosion damage was the use of relatively high-velocity mercury flow to disrupt the cavitation bubble growth and collapse process. Experiments performed at the Los Alamos National Laboratory (LANL) Weapons Nuclear Research 
(WNR) facility in 2005 demonstrated the mitigating effect of flow on erosion damage, where results from in-beam experiments showed that flow reduced the damage on specimens by half compared to specimens that were exposed to similar conditions in stagnant mercury [10]. Subsequent experiments verified that flow was "somewhat effective" at reducing erosion damage, but results indicated that flow was less effective at reducing damage compared to other mitigating techniques investigated [11].

A modified SNS target design was developed to exploit the mitigation effect of flow observed during the WNR experiments, called the jet-flow target design. Mercury flow pathways in the jet-flow target design were reconfigured to create a high-velocity layer of flowing mercury against the mercury vessel inner wall at the beam entrance region where the majority of the erosion damage occurs, as shown in Figure 6. High-velocity flow against the inner window was thought to disrupt the bubble growth and collapse process, which would protect the inner wall from erosion damage.

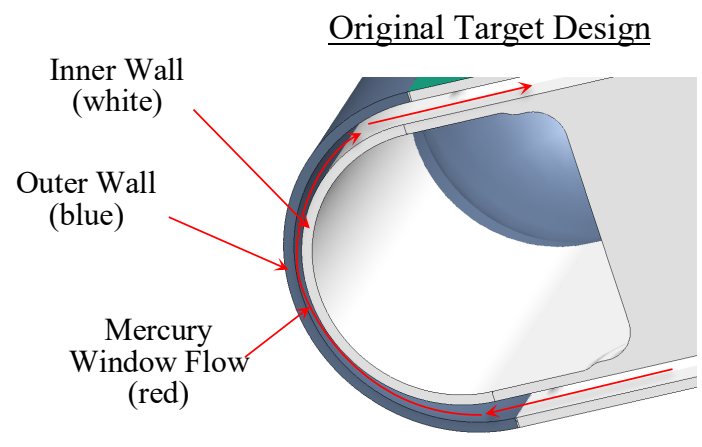

(a)

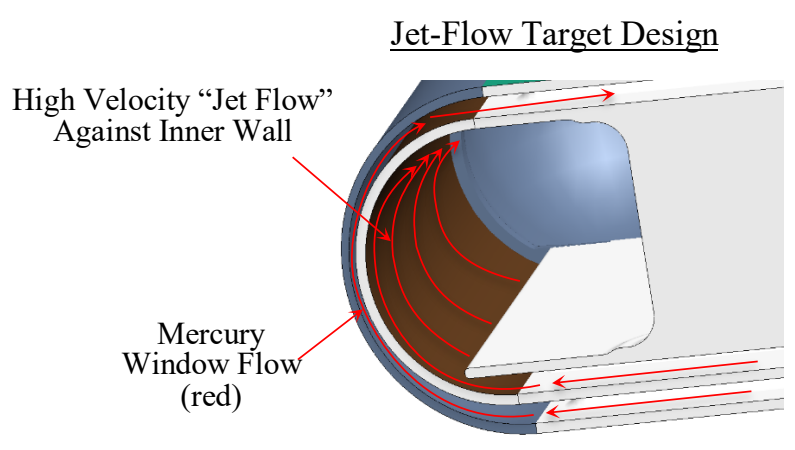

(b)

Figure 6. Side views of the beam entrance region of the (a) original and (b) jet-flow target designs.

Target 10 was the first jet-flow target operated at the SNS, but unfortunately a mercury leak occurred relatively early during operation due to failure of a weld joining the front and transition sections of the mercury vessel near the middle region of the target. However, the early failure provided valuable insight into the progression of damage to the inner wall at the beam entrance region of the original and jetflow target designs. Coincidentally, an earlier original design target, Target 6 , also leaked at a weld after operating at almost the identical average power and to the same total absorbed energy as Target 10 . The PIE characterization of these two targets provided a direct comparison of the damage observed in original and jet-flow targets operated under almost identical conditions. Images of samples from the inner walls from Targets 6 and 10 are shown in Figure 7. Erosion damage to the Target 10 inner wall was less severe at the center compared to Target 6 , with no $\Phi$-shaped damage pattern observed on the Target 10 inner wall. Small patches of erosion were observed near the center of the Target 10 inner wall, but the wall was not perforated or fractured like the Target 6 inner wall. These initial observations of a jet-flow target inner wall were encouraging and additional jet-flow targets were fabricated and operated to further investigate the mitigating effect of flow at the beam entrance inner wall. 

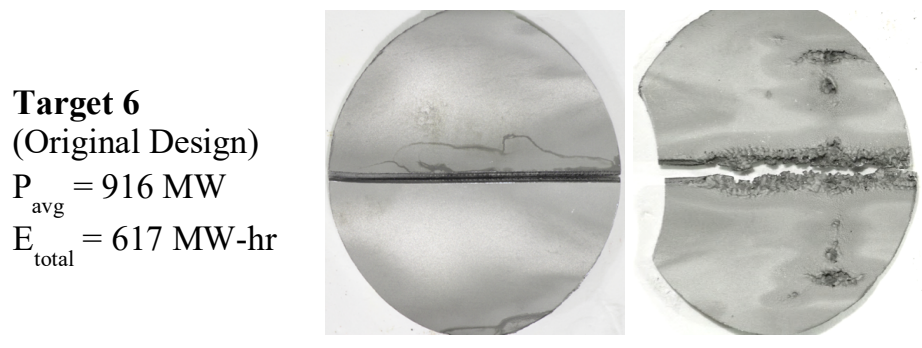

$$
\begin{aligned}
& \text { Target 10 } \\
& \text { (Jet-flow Design) } \\
& \mathrm{P}_{\text {avg }}=1052 \mathrm{MW} \\
& \mathrm{E}_{\text {total }}=601 \mathrm{MW}-\mathrm{hr}
\end{aligned}
$$
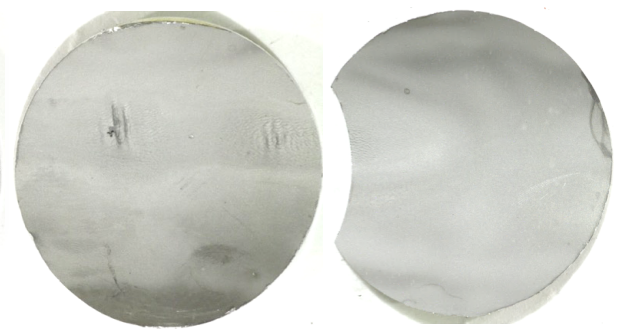

Figure 7. Photographs of specimens from the inner wall of Target 6 (original design) and Target 10 (jet-flow design).

Only original design targets were available for operation after the Target 10 leak occurred, and the next target, Target 11, leaked shortly after beginning operation at the same weld location that caused leaks in Targets 6 and 7. The Target 11 leak occurred at a weld on a trapezoidal shaped plate on the bottom of the target vessel designed to enclose the window flow supply plenum, which is identified in Figure 3 . The design and fabrication method were revised to eliminate the trapezoidal cover plate on all subsequent SNS targets.

\subsubsection{EROSION-INDUCED LEAKS IN TARGETS 12 AND 13}

Following the Target 11 leak, Target 12 was operated for an extraordinarily long time, setting the current record for total absorbed energy in an SNS target at 4,460 MW-hr. Unfortunately, Target 12 leaked during operation and required an unplanned target change that interrupted the neutron-science user program. PIE techniques were used to locate the leak, and the area of the vessel containing the leak was sampled using the target sampler. The Target 12 leak was located on the single wall section on the driver side of the inner wall (refer to Figure 2), as shown in Figure 8. The erosion damage pattern on the sample from the passenger side of the inner wall, designated Disk 17, was almost an identical mirror image of the damage on the driver side, but no leak occurred on the passenger side. 


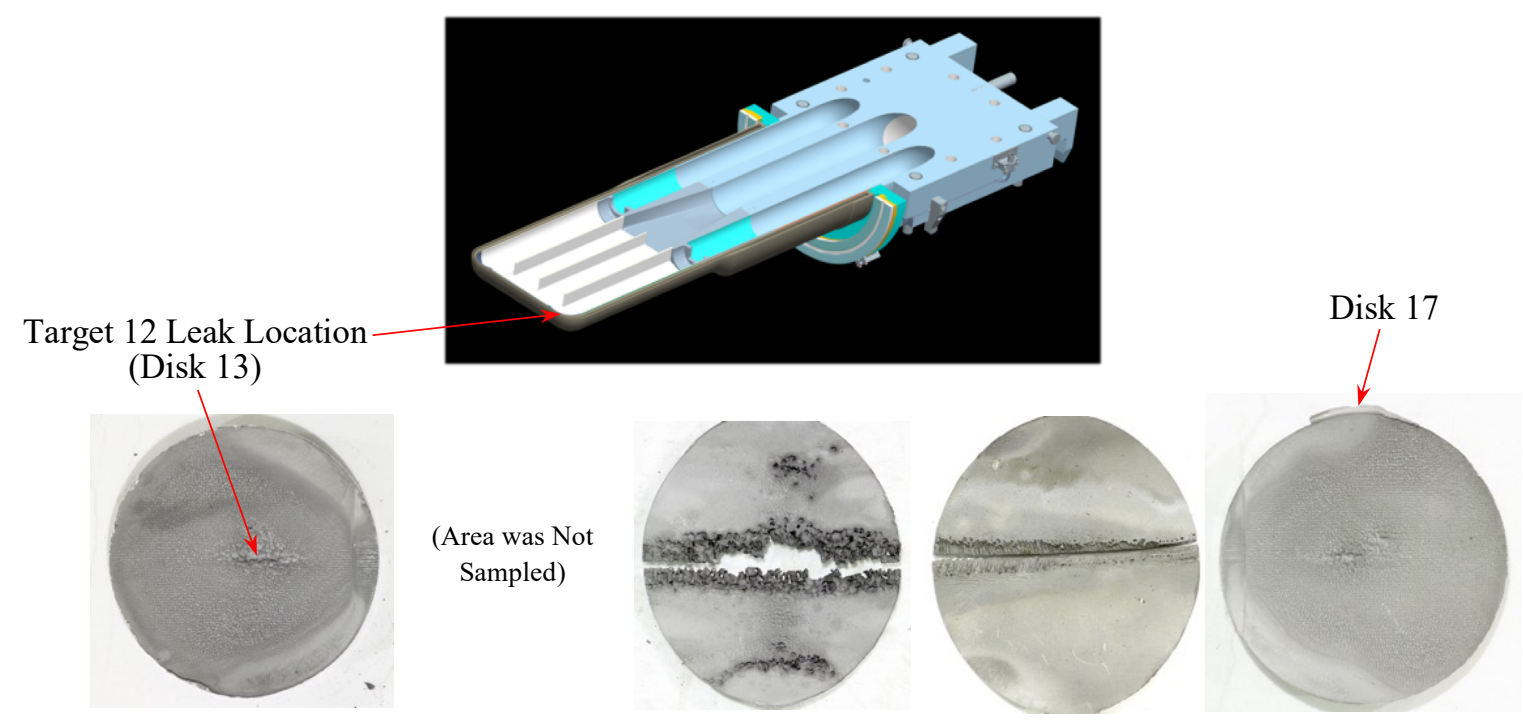

Figure 8. Photograph mosaic of samples from the inner wall of Target 12 and a diagram identifying the leak location.

Subsequent photography showed the hole through the Target 12 inner wall was very small and located at the base of the eroded cavity, as shown in Figure 9. The formation of this cavity appeared to be caused by mass-wastage type of erosion, which is described in greater detail in reference [5]. This type of erosion occurs when an erosion pit penetrates the hardened Kolsterising ${ }^{\circledR}$ treated layer on the vessel surface and begins to erode the soft underlying base material. As erosion continues, sections containing the hardened surface layer are undercut by erosion of the soft underlying material and the sections begin to lose support. After the underlying material is sufficiently eroded the sections of the hardened surface layer break loose from the surface and become entrained in the mercury flow. Flow is known to mitigate cavitationinduced erosion damage [10], but as the cavity grows, the mitigating effect of flow is reduced. The reduction in flow mitigation is expected to exacerbate the cavity growth process and accelerate localized erosion damage. 


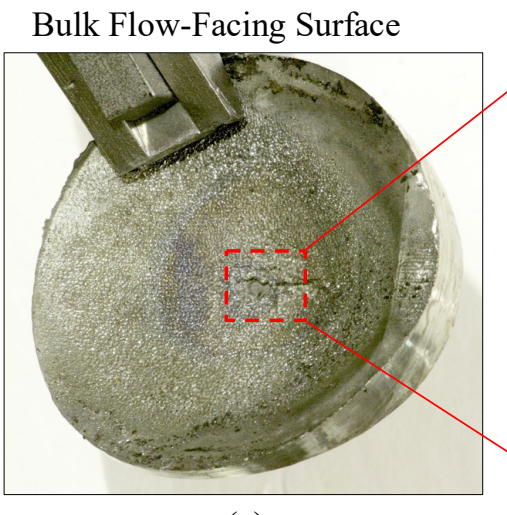

(a)

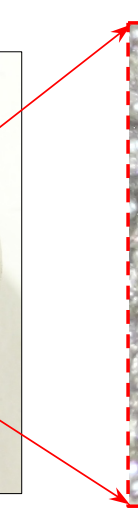

$1-2 .-1-1-1$

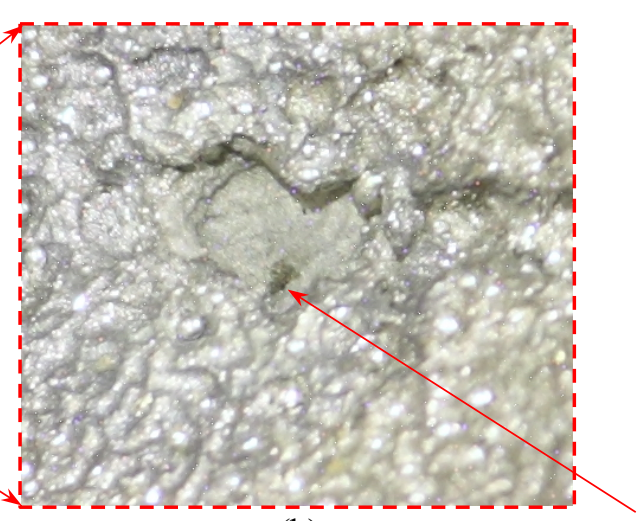

(b)

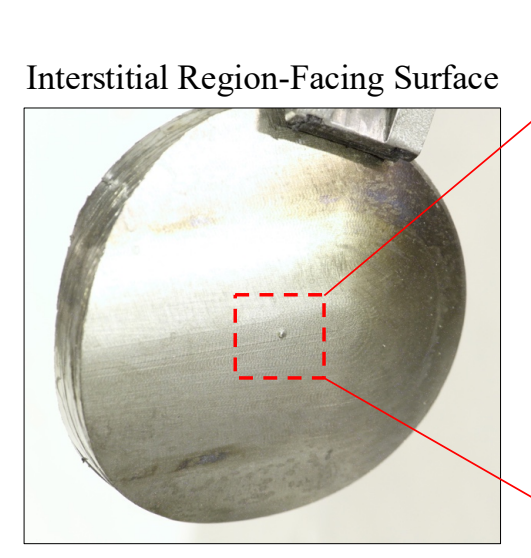

(c)

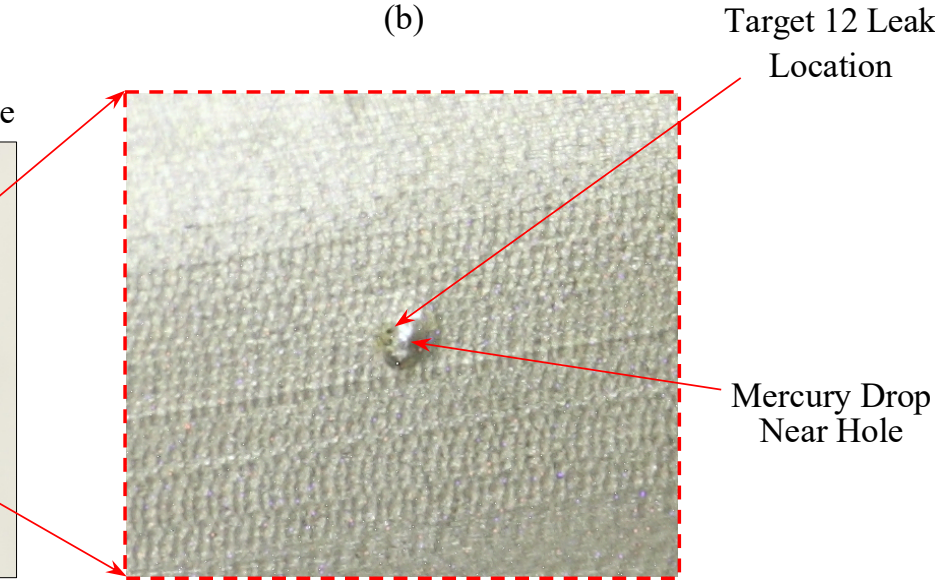

(d)

Figure 9. Photographs of the sample containing the Target 12 leak location: (a) bulk-flow facing surface, (b) magnified image of the leak location, (c) interstitial region-facing surface, and (d) a magnified image of the leak location viewed from the interstitial region-facing side. NOTE: a drop of mercury is shown adhering to the surface near the leak location hole in (d).

After Target 12 was removed from service, Target 13 was operated to 2,589 MW-hr and subsequently developed a leak. PIE inspection with an articulating videoprobe revealed that the Target 13 leak had occurred at the same location as the Target 12 leak: in the single-wall section of the inner window at the end of the bulk-flow supply passage on the driver side, as shown in Figure 10. Also, similar to Target 12 , the erosion pattern on the passenger side of Target 13 was a mirror image of the erosion pattern on the side that leaked. But unlike Target 12, the shape of the erosion pattern at the center of the inner window of Target 13 did not have a pronounced "I" component of the $\Phi$-shape pattern previously observed, rather the pattern on the Target 13 inner window contained more large pits located at the center compared to the Target 12 window. 


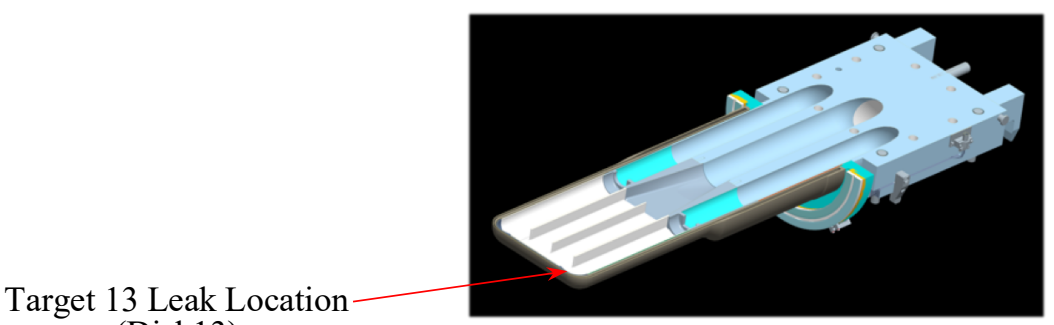

(Disk13)
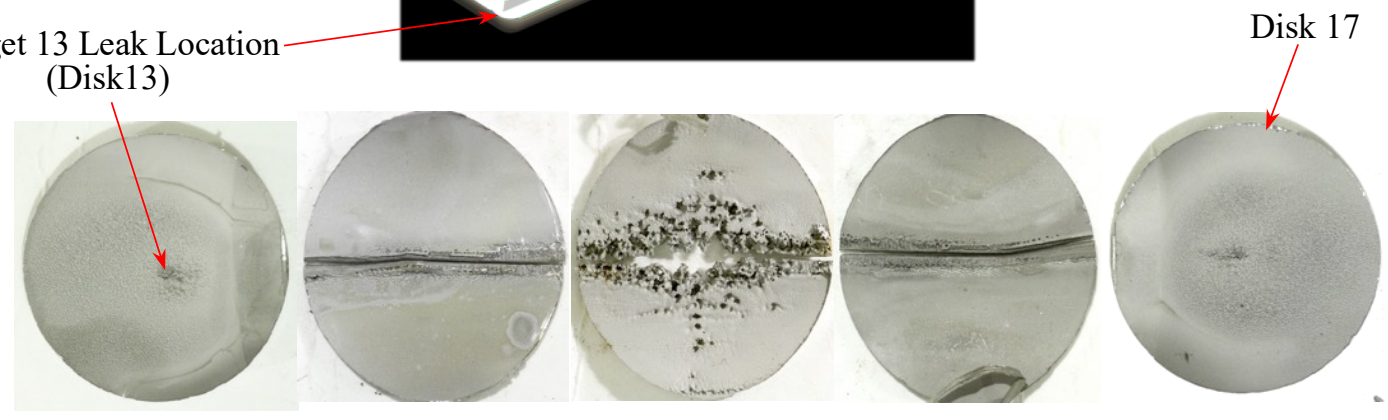

Figure 10. Photograph mosaic of samples from the inner wall of Target 13 and a diagram identifying the leak location.

\subsubsection{CHARACTERIZATION OF CAVITATION-INDUCED EROSION DAMAGE}

After the Target 13 leak was discovered it was acknowledged that cavitation-induced erosion at the ends of the supply passages was a vulnerability to the mercury vessel. In response to the Targets 12 and 13 leaks a plan was established to: (1) develop a relationship between operating power and the extent of erosion at the leak locations and (2) create a reference set of erosion data for comparison with future targets. The relationship between operating power and the extent of erosion was desired to facilitate decision making on the operating cycles at the SNS. Concurrent with PIE characterizations of SNS target modules, the target design is continually modified and improved for successive targets to address issues identified during manufacturing and PIE. A method to measure and correlate erosion damage relative to operating history was necessary to quantitatively evaluate target design changes and determine if changes to the target design reduced erosion damage.

Photography of the samples removed from the beam entrance region allow qualitative characterization of the erosion damage to the target vessel, but does not provide quantitative data required to understand the relationship between operating history and erosion damage. A 3-dimensional characterization method called laser line scanning was identified as a potential technique to quantitatively measure erosion damage to SNS target samples. Laser line scanning is a non-contact characterization technique capable of directly measuring the 3-dimensional topography and shape of an object. The laser line scanning process creates a high resolution 3-dimensional point cloud of an object by incorporating triangulation-based measurements from a laser line emitter/detector with position data from encoders on the arm supporting the laser scanner. After scanning, the point cloud data can be interrogated and analyzed to calculate the amount of material removed from the vessel surface and the thickness of material remaining at the erosion location. 


\subsubsection{Implementation OF The TARget Management Plan}

While laser line scanning was identified as a viable method to measure erosion damage, the operational nature of the SNS complicates the correlation of operational history and erosion behavior. SNS Targets 1-13 were operated at appreciably different power levels throughout their lifetimes. Since the erosion rate is highly dependent on operational power [12], the different operational power levels experienced by each target greatly complicated correlation of the erosion observations and operational power levels. To better understand the relationship between operational power level and erosion rate at the location of the Target 12 and 13 leaks, a plan was developed to operate targets at different fixed power levels throughout their lifetimes and measure the erosion observed at the leak locations after service. Operating targets at a near-constant power level throughout their lifetime allows direct comparison of erosion rates measured from different targets that were operated at different power levels. The plan was articulated in a document titled Target Management Plan (TMP) [13], which was also intended to facilitate target inventory and fabrication management.

The TMP was implemented following the Target 13 leak; Target 14 was the first target operated at a near-constant power level throughout most of its lifetime. Target 14 was operated at approximately 1.0 MW to provide a moderate-power baseline for the erosion damage characterization measurements, while Target 15 was operated at approximately 1.1 MW to provide data for an incrementally higher power level. Targets 14 and 15 were both original target designs and did not feature any cavitation mitigation techniques, other than the Kolsterising ${ }^{\circledR}$ treatment. Both targets were successfully operated to total absorbed energy levels of 2,762 and 1,667 MW-hr, respectively, without leaks.

\subsubsection{OBSERVATIONS OF DAMAGE IN High-USE JET-FLOW TARGETS}

Targets 16 and 17 were both jet-flow targets and provided information on damage to jet-flow targets operated at average power and total absorbed energy levels of 968 and 1,127 MW, and 1,780 and 1,936 MW-hr, respectively. While the Target 10 results showed the jet-flow design had reduced the damage to the inner wall compared to Target 6, PIE of Targets 16 and 17 revealed that appreciable damage occurred to the inner wall during operation. Photographs of specimens from the inner walls of Targets 16 and 17 are shown in Figure 11. Unlike Target 10, the inner walls of Targets 16 and 17 were fractured along the horizontal centerline and an appreciable amount of erosion damage was present at the center of the beam entrance region. These observations showed that erosion damage mitigation with flow was not effective at protecting the inner wall from erosion or fracture, and alternative mitigation techniques would be necessary to protect the mercury vessel inner wall. 
Target 16

(Jet-flow design)

$\mathrm{P}_{\mathrm{avg}}=968 \mathrm{~kW}$

$\mathrm{E}_{\text {total }}=1780 \mathrm{MW}-\mathrm{hr}$
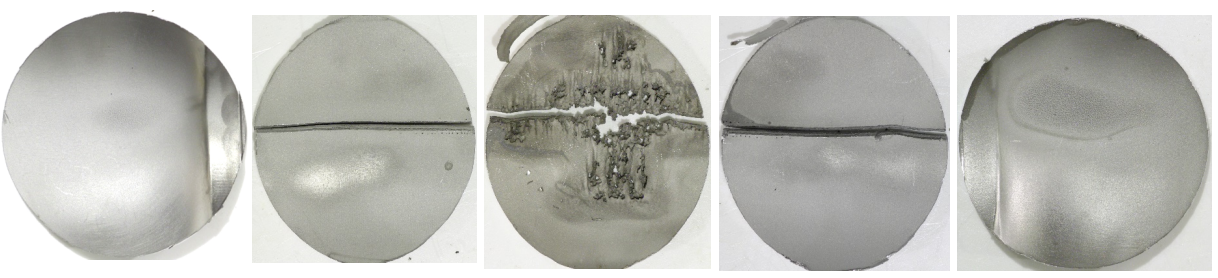

\section{Target 17}

(Jet-flow design)

$\mathrm{P}_{\text {avg }}=1127 \mathrm{~kW}$

$\mathrm{E}_{\text {total }}=1936 \mathrm{MW}-\mathrm{hr}$
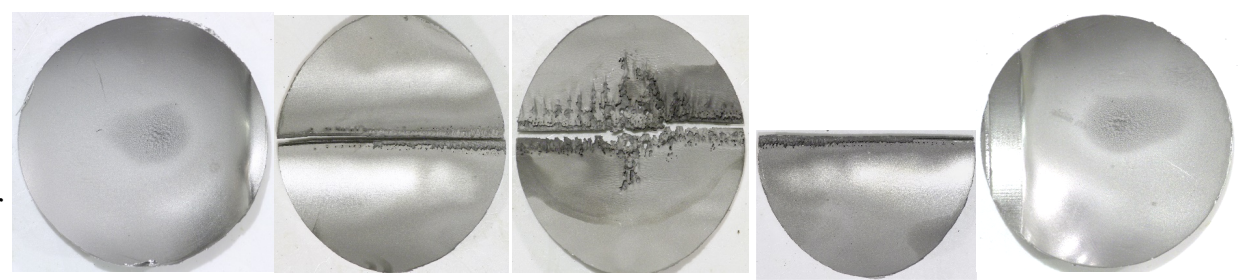

Figure 11. Photographs of specimens from the inner wall of Targets 16 and 17 (jet-flow designs) after operation.

\subsubsection{IMPLEMENTATION OF SMALL-BUBBLE GAS INJECTION}

PIE characterizations have shown that both hardening of the mercury-facing surfaces of the mercury target vessel and the presence of a jet flow against the inner wall appear to delay the onset of steady-state cavitation-induced erosion, but they do not affect the bubble formation and collapse throughout the target volume. Another erosion mitigation technique potentially capable of reducing erosion damage throughout an SNS target is called small-bubble gas injection. Small-bubble gas injection introduces a high number density of small diameter $(<150 \mu \mathrm{m})$ helium bubbles into the flowing mercury. Results from WNR experiments showed erosion damage on specimens tested with small-bubble gas injection was four times lower compared to erosion damage on specimens tested in stagnant mercury [10]. Small-bubble gas injection also decreased the stress and strain in the walls of target containers tested at WNR [10, 12]. Also, recent experience operating SNS targets in situ with stain sensors and small-bubble gas injection show the strain response of the mercury target vessel was greatly reduced with gas injection [14].

Targets 18 and 19 were both jet-flow target designs that were operated with small-bubble gas injection. The gas flow rate for the Target 18 bubbler varied throughout operation from 0.25 to 0.45 standard liters per minute (SLPM), whereas the gas flow rate during Target 19 operation was fairly constant at approximately 0.45 SLPM. Photography of samples from Targets 18 and 19 show an appreciable decrease in erosion damage compared to previous targets, as shown in Figure 12, and the inner walls were not fractured. The most pronounced erosion occurred at the center of the window, where the erosion pattern had an elongated morphology oriented parallel to the direction of the high-velocity jet flow. Erosion damage to the Disk 13 position in Targets 18 and 19, where Targets 12 and 13 leaked, was not appreciable compared to previous targets. 


\section{Target 18}

(Jet-flow design)

$\mathrm{Q}_{\text {avg }}=0.45-0.25$ SLPM

$\mathrm{P}_{\text {avg }}=1128 \mathrm{~kW}$

$\mathrm{E}_{\text {total }}=1261 \mathrm{MW}-\mathrm{hr}$
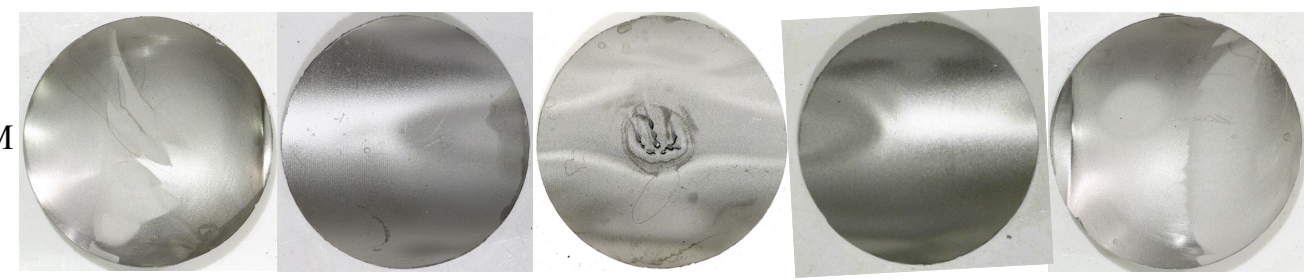

\section{Target 19}

(Jet-flow design)

$\mathrm{Q}_{\text {avg }}=\sim 0.45 \mathrm{SLPM}$

$\mathrm{P}_{\mathrm{avg}}=1207 \mathrm{~kW}$

$\mathrm{E}_{\text {total }}=1987 \mathrm{MW}-\mathrm{hr}$

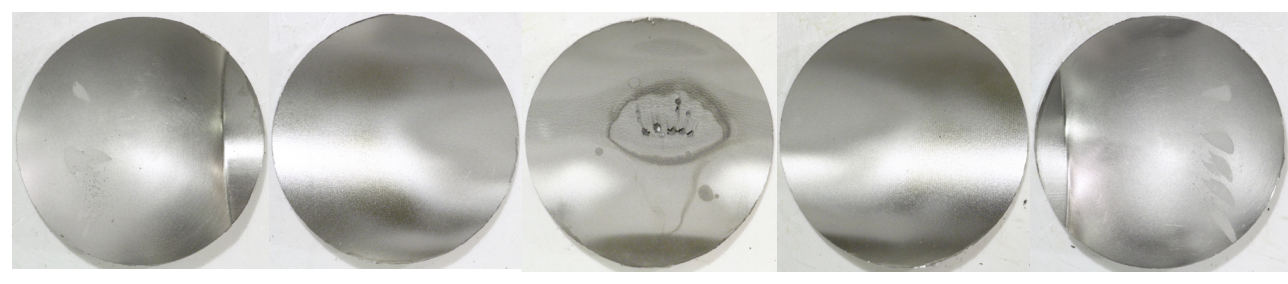

Figure 12. Photographs of specimens from the inner wall of the Targets 18 and 19 (jet-flow designs) after operation with small-bubble gas injection.

The preceding discussion described the general history of recent SNS target development and implementation of cavitation-induced erosion mitigation techniques deployed at the SNS. The following report describes and evaluates results from laser line scan characterization of select SNS target samples from Targets 10-19.

\section{EXPERIMENTAL Procedure}

\subsection{LASER LINE SCANNING SYSTEM}

A laser line scanner produced by $\mathrm{FARO}^{\circledR}$ was utilized to scan samples from SNS target vessels; the model used for the scans was a FARO ${ }^{\circledR}$ Edge ScanArm ${ }^{\circledR}$ HD, shown in Figure 13. This model had an accuracy of \pm 0.001 in $( \pm 25.4 \mu \mathrm{m})$ and a scan rate of 280 frames per second, which corresponds to a scan rate of 560,000 points per second. The scanner was calibrated per the manufacturer specifications prior to scanning the target specimens and the scanner was operated in "high accuracy" mode. Geomagic ${ }^{\circledR}$ DesignX software was used for data acquisition during the scans, and post-scan modeling and analysis were performed using Geomagic ${ }^{\circledR}$ Design Direct and Geomagic ${ }^{\circledR}$ Control software packages. 


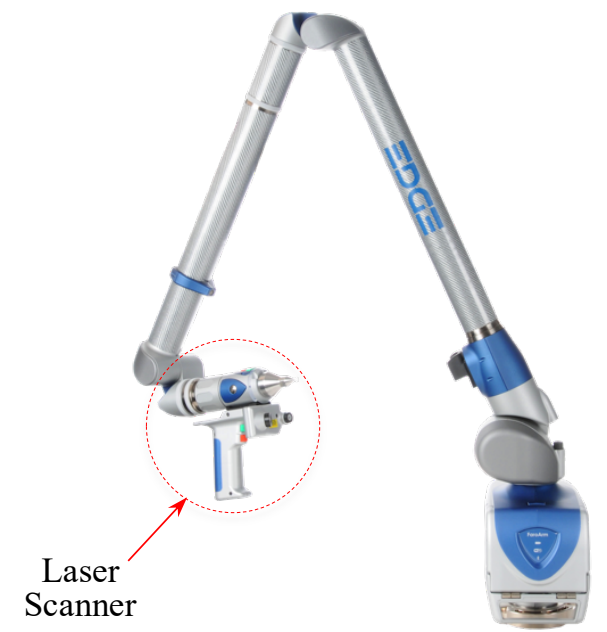

(a)

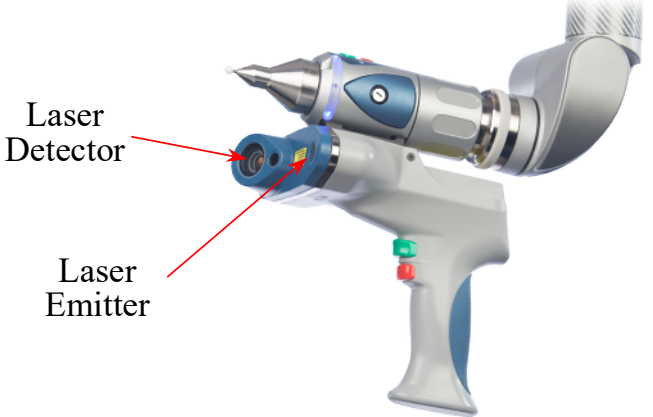

(b)

Figure 13. Images of the $\mathrm{FARO}{ }^{\circledR}$ Edge ScanArm ${ }^{\circledR} \mathrm{HD}$ laser line scanner: (a) arm/scanner assembly and (b) close-up image of laser scanner (Photographs courtesy of FARO ${ }^{\circledR}$ Technologies Inc.).

The FARO ${ }^{\circledR}$ Edge ScanArm ${ }^{\circledR}$ HD laser-line scanner was designed for hands-on operation, which presents a challenge when scanning highly radioactive samples. The distance between the scanner and the object must be held within an optimal range and the operator must have a clear view of the object during the scan to ensure all sections of the disk specimen are scanned. But one significant advantage to the laser scanning technique is the minimal time required to perform a scan. Mockup scanning trials with a prototypic disk specimen showed that approximately one minute was needed to properly scan two sides of one disk. Due to the complications with performing the scans remotely and the short scan time required to perform a measurement, the decision was made to perform the first series of scans manually. A technician was selected to perform the scans and extensive mockup testing was performed to verify the assumptions in the "as low as reasonably achievable" (ALARA) calculations were valid.

The positions of the scanning arm and the specimen must remain fixed relative to each another during the scanning process, and the specimen must be remotely mounted prior to the scan. A specimen holder and base-support structure were designed to facilitate remote handling of the disk specimens for loading and hands-on scanning using the $\mathrm{FARO}^{\circledR}$ arm, as shown in Figure 14. The base structure is designed to securely fix the positions of the $\mathrm{FARO}^{\circledR}$ arm and disk specimen during the scanning process. A specimen mount was designed to permit remote loading of a disk specimen and securely hold the disk specimen during scanning; the mount was secured to the base-support assembly via a remotely actuated toggle clamp. Two specimen mounts were utilized to quickly change specimens and decrease the time between scans when technicians were outfitted in cumbersome protective clothing. 


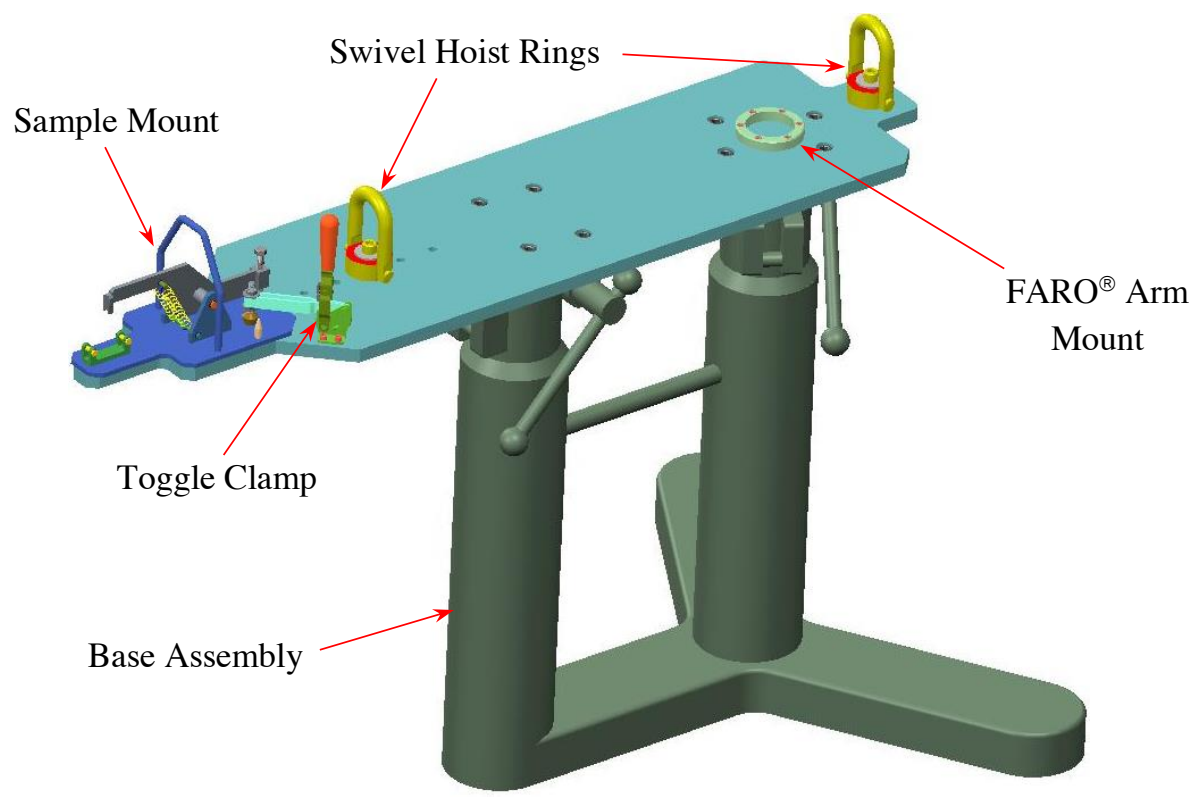

(a)

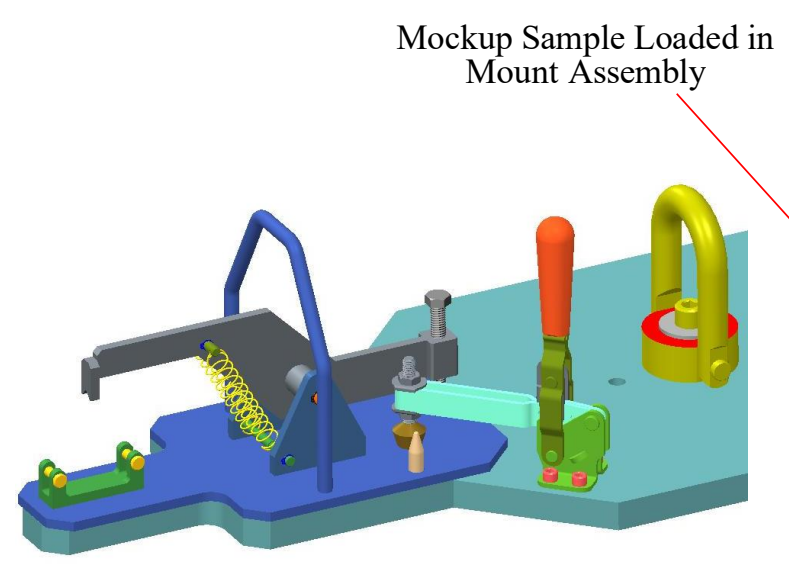

(b)

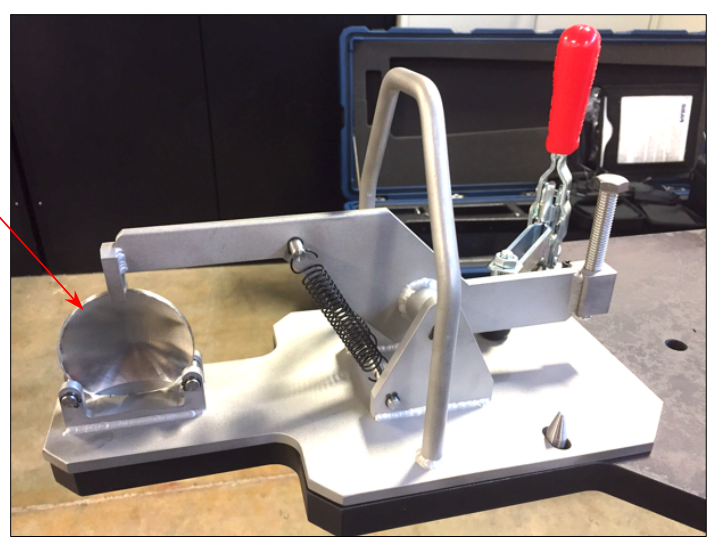

(c)

Figure 14. Schematics of (a) the sample base-support assembly and (b) sample mount for the FARO ${ }^{\circledR}$ laser line scan characterizations, and (c) picture of mockup specimen loaded in mount.

After the specimen base and mounts were fabricated, extensive mockup trial scans were performed to confirm the scanning procedure was properly evaluated to ensure minimal exposure to personnel performing the scans. Scanning two disks in one operation was desired to maximize productivity of service cell operations. Prior to introducing specimens into the transfer cell, where the scans were performed, the base assembly was set up in the transfer cell, the FARO ${ }^{\circledR}$ arm was mounted to the base assembly, and cables were connected from the $\mathrm{FARO}^{\circledR}$ arm to the data acquisition computer outside the cell. After the system was set up in the transfer cell the room was evacuated; samples were brought into the transfer cell and loaded into the sample holders, and a sample holder containing a specimen was secured to the base structure. After the sample was secure and the computer was communicating with the scanner, a technician entered the transfer cell, picked up the $\mathrm{FARO}^{\circledR}$ arm scanner, and scanned one side of the disk. After scanning the first side of the sample, the data acquisition program was reset for the second side by personnel operating 
the data acquisition computer, requiring a 10-second delay between scans. After scanning the second side of the disk the technician quickly exited the room. After the first disk was scanned the second mount was secured to the base structure and the scanning procedure was repeated. Nine disk samples from five different targets were scanned using the manual operation method.

It was recognized during early mockup testing that manually scanning disk samples was not ideal because it required the technician performing the scanning to be exposed to appreciable dose rates, and another scanning method was developed to remove the technician from the scanning process. The feasibility of using the through-the-wall manual manipulators was considered but was deemed impractical due to the limited reach and articulation of the manipulators. After researching viable options, it was determined that a programable robotic system would be the best option for remote laser line scanning of SNS target samples.

An industrial robot arm was procured from Universal Robots to perform the laser line scans; the UR10 model, shown in Figure 15, was selected due to the 51.2-inch reach and 22-pound load capacity. An interface adapter between the robot arm and the scanner handle was designed and fabricated using a polymer additive manufacturing system. While the UR10 was capable of the performing the same movements required to scan SNS target specimens with the FARO ${ }^{\circledR}$ Edge ScanArm ${ }^{\circledR}$ HD scanner, the UR10 could not activate a button on the scanner handle that must be pressed prior to a scan. Therefore, a procedure was developed to press the scanner button using a through-the-wall manipulator just prior to performing a scanning operation. The movement pathways for each scan were programmed during mockup testing to scan both sides of SNS samples from different angles to fully scan the recessed eroded cavities present on some samples. The robot was operated and data acquired by a touch-screen controller and computer, respectively, located outside the transfer cell, which completely eliminated dose exposure to personnel during the scans. Prior to deployment of the UR10 a total dose of $160 \mathrm{mrem}(1.6 \mathrm{mSv})$ was received by the one technician to scan 9 samples manually, and after the UR10 was implemented a total dose of 42 mrem $(0.42 \mathrm{mSv})$ was received by 7 people to scan 19 samples.

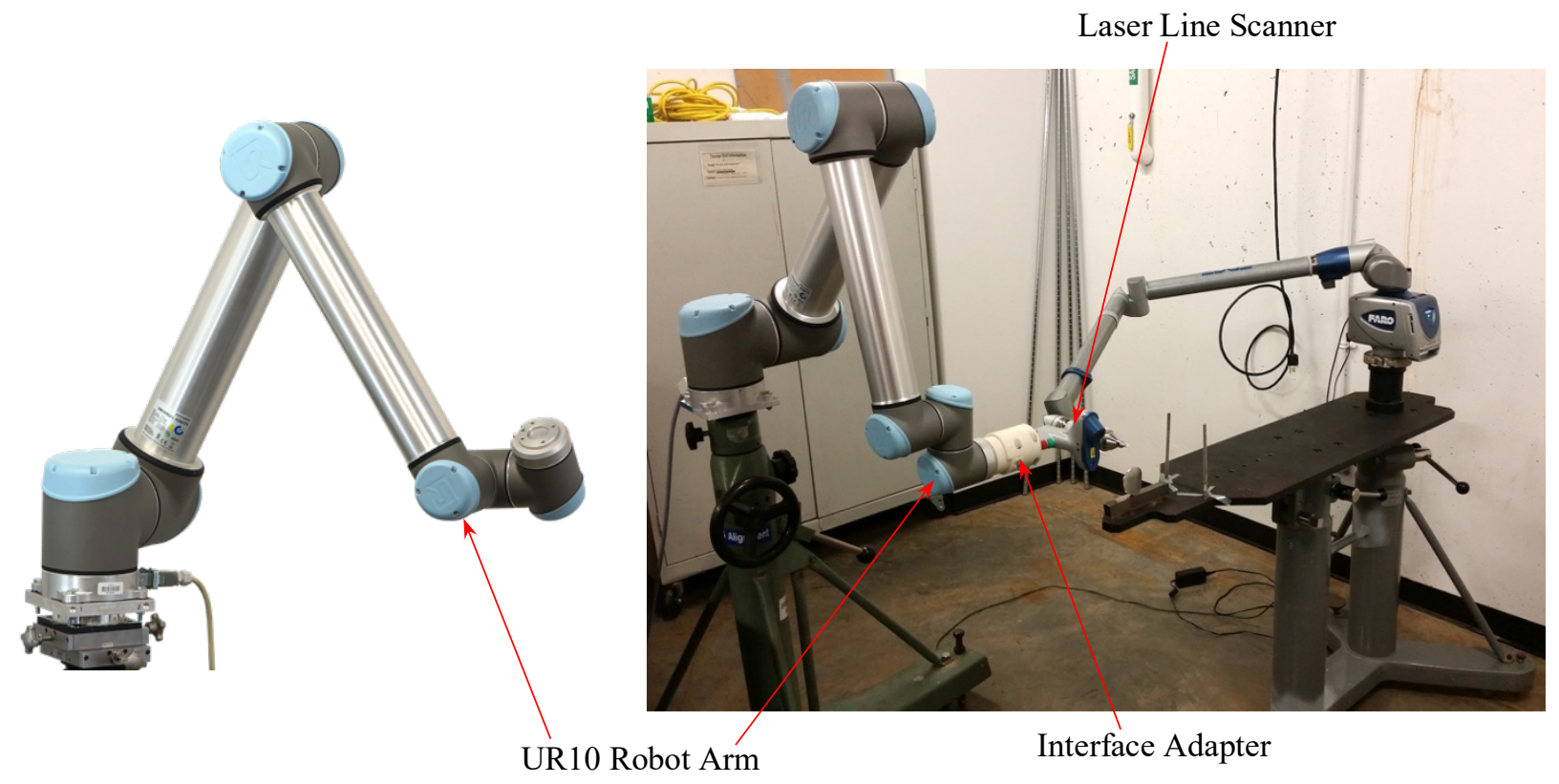

Figure 15. Images of the UR10 robot arm and laser line scanning system developed to remotely scan target samples. 


\subsection{DAta Analysis}

Following the scans, the data sets were analyzed to calculate various metrics used to characterize cavitation-induced erosion to the disk samples. The primary metrics of interest were: (1) depth of material eroded from the disk surface, (2) the disk thickness, (3) the location of the minimal thickness or hole, and (4) the mean depth of erosion. The amount of material eroded from the thickness of each disk was calculated by comparing the point cloud produced by the scan to a computer model of an ideal (non-eroded) sample from an SNS target vessel. The disk thickness values across the sample were calculated using the scan point cloud data. The location of the minimal thickness or hole was calculated relative to a common coordinate system so the locations of the erosion cavities/holes could be compared to one another. The mean depth of erosion was calculated for $1 \mathrm{~cm}$ and $2 \mathrm{~cm}$ diameter circles by dividing the volume of material removed from the $1 \mathrm{~cm}$ and $2 \mathrm{~cm}$ circles centered over the maximum pit/cavity depth by the area of the $1 \mathrm{~cm}$ and 2 $\mathrm{cm}$ circles.

Samples from four different locations (see Figure 5 for sample identification scheme) of the mercury target vessel were scanned:

1. the single wall section at the end of the "driver" side mercury supply passage (Disks 13)

2. the single wall section at the end of the "passenger" side mercury supply passage (Disks 17)

3. the bulk-flow facing surface of the inner wall from the center cut (Disk 5)

4. the window-flow facing surface of the outer wall from the center cut (Disk 6)

Each location required a different data analysis procedure due to the different geometries of the samples and the Disk 13 and 17 samples of different targets required modifications due to design changes that were made to later target vessels at these locations.

To compute these erosion metrics, a standard coordinate system definition was established for each type of disk. These multiple definitions are required since the disk geometry has varied among the various target designs. The coordinate system definitions are explained in Figures 16 through 19. These figures show the geometry of the target nose in the area from which the samples were removed. The colors represent surfaces which correspond to different geometry primitives.

Taking advantage of the symmetry of the targets, the same coordinate system definition was used for both the driver- and passenger-side disks of each type of target. But since the geometry of the two sides of the target is mirrored, the coordinate systems for the two disks are rotated with respect to each other when viewed in the context of the whole target. That is, for the driver side specimen (Disk 13), $+\mathrm{Y}$ is upwards relative to the target module, while for the passenger side specimen (Disk 17), $+Y$ is down. And the $+\mathrm{X}$ axis points towards the center plane of the target module in both disk coordinate systems. 
The coordinate system used to analyze the scan data for Disks 13 and 17 from Target 12, shown in Figure 16, was fitted to the scan points by finding three translations and three rotations, simultaneously, that optimized these four conditions:

1. The points on the undamaged portion of the red surface lie at a distance of 1.93 in from the origin

2. The points on the undamaged portion of the green surface lie at a distance of 1.93 in from the $\mathrm{X}$-axis

3. The points on the undamaged portion of the blue surface lie at a distance of 1.93 in from the point on the $\mathrm{X}$-axis where $\mathrm{X}=0.395$ in

4. The points on the undamaged portion of the yellow surface lie at a distance of 1.57 in from a line in the $X-Z$ plane where $Z=-0.160$ in
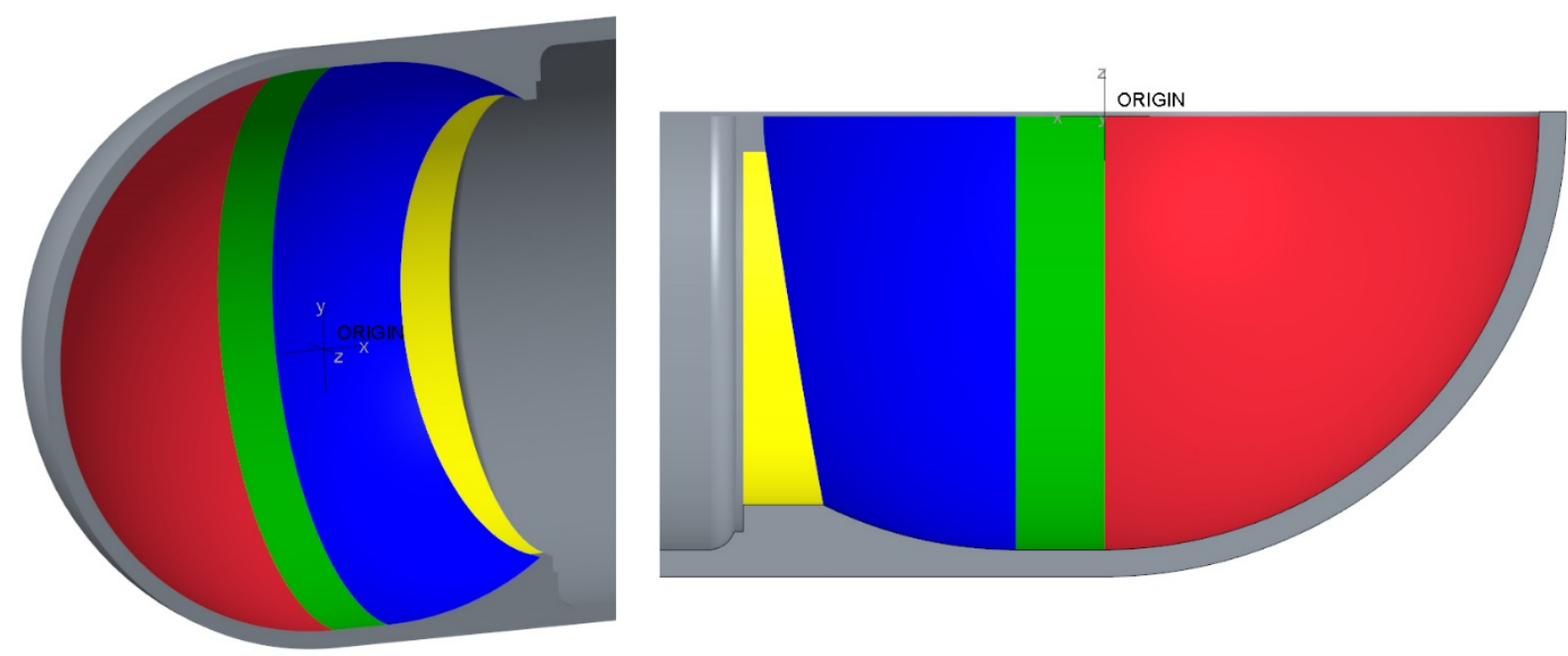

Figure 16. Illustration of the coordinate system definition for Disks 13 and 17 from Target 12 .

The coordinate system used to analyze the scan data for Disks 13 and 17 from Targets 13-15, shown in Figure 17, was fitted to the scan points by finding three translations and three rotations, simultaneously, that optimized these four conditions:

1. The points on the undamaged portion of the red surface lie at a distance of 1.93 inches from the origin

2. The points on the undamaged portion of the green surface lie at a distance of 1.93 inches from the $\mathrm{X}$ axis

3. The points on the undamaged portion of the blue surface lie at a distance of 1.93 inches from the point on the $\mathrm{X}$-axis where $\mathrm{X}=0.395$ inch

4. The points on the undamaged portion of the yellow surface lie at a distance of 1.57 inches from a line in the $\mathrm{X}-\mathrm{Z}$ plane where $\mathrm{Z}=-0.190$ inch 

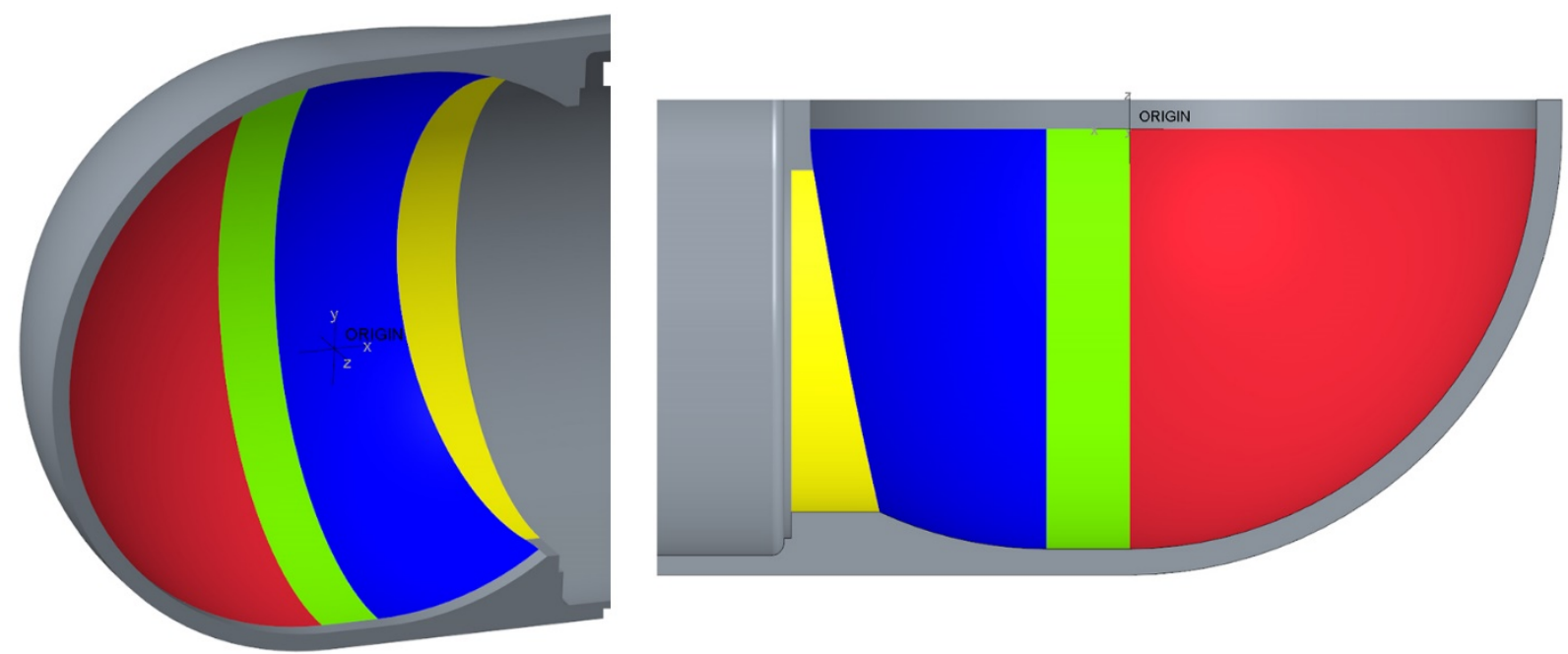

Figure 17. Illustration of the coordinate system definition for Disks 13 and 17 from Targets 13, 14, and 15.

The coordinate system used to analyze the scan data for Disks 13 and 17 from Targets 16 and 17, shown in Figure 18, was fitted to the scan points by finding three translations and three rotations, simultaneously, that optimized these five conditions:

1. The points on the undamaged portion of the red surface lie at a distance of 1.93 inches from the origin.

2. The points on the undamaged portion of the green surface lie at a distance of 1.93 inches from the Xaxis.

3. The points on the undamaged portion of the blue surface lie at a distance of 1.93 inches from the point on the $\mathrm{X}$-axis where $\mathrm{X}=0.328$ inch.

4. The points on the undamaged portion of the white surface lie on the surface of a torus having a major radius of $1.210 \mathrm{inch}$ and a minor radius of $0.620 \mathrm{inch}$. This torus is centered at the point on the Xaxis where $\mathrm{X}=0.831 \mathrm{inch}$, and the torus is oriented so that it is radially symmetric about the $\mathrm{X}$-axis.

5. The points on the undamaged portion of the yellow surface lie at a distance of 1.435 inches from a line in the $\mathrm{X}-\mathrm{Z}$ plane where $\mathrm{Z}=-0.190$ inch. 

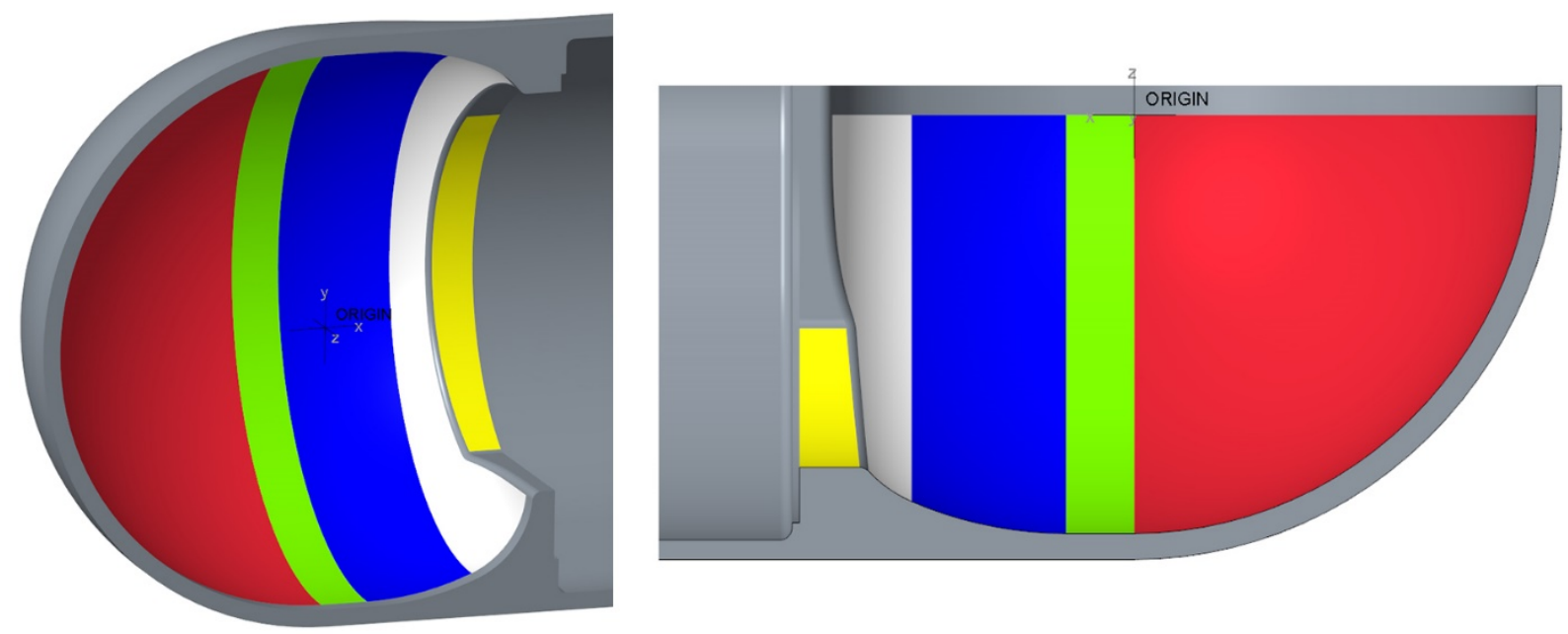

Figure 18. Illustration of the coordinate system definition for Disks 13 and 17 from Targets 16 and 17.

For disks 13 and 17 of Targets 18 and 19, a different approach was taken to fitting the coordinate system to the scan data. Photographs showed that very little erosion had occurred, and it was limited to one region of the interior surface. To obtain the most accurate fit in the eroded region, several other regions of the interior surface were ignored in the fitting process. So, the coordinate system used to analyze the scan data, shown in Figure 19, was fitted to the scan points by finding three translations and three rotations, simultaneously, that optimized these four conditions:

1. The points on the undamaged portion of the red surface lie at a distance of 1.93 inches from the origin

2. The points on the undamaged portion of the green surface lie at a distance of 1.93 inches from the Xaxis

3. The points on the undamaged portion of the yellow surface lie at a distance of 1.435 inches from a line in the $\mathrm{X}-\mathrm{Z}$ plane where $\mathrm{Z}=-0.270$ inch

4. The points on the undamaged portion of the orange surface lie at a distance of 2.05 inch from a line in the $X-Z$ plane where $Z=-0.080$ inch 

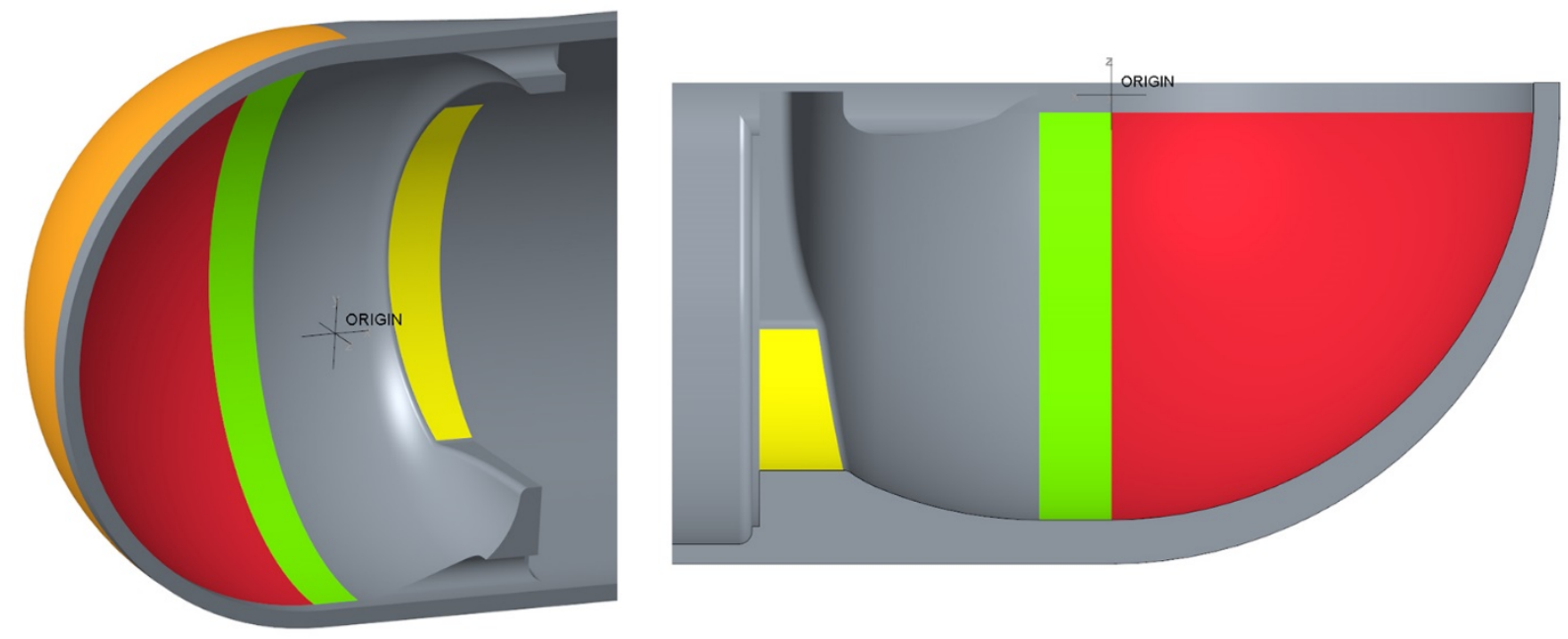

Figure 19. Illustration of the coordinate system definition used to calculate erosion metrics for Disks 13 and 17 from Targets 18 and 19.

Disks 5 and 6 were removed from the inner and outer wall at the center of the target module (see Figure 5). Since, these disks were removed from a portion of the target with simple cylindrical geometry for both the interior and exterior surfaces, it was not possible to completely define a coordinate system related to the target model. Also, since the exact location of the sample cut varies between each sampling cut, coordinate systems between specimens from different targets could not be established. Therefore, no point of maximum erosion was calculated for the Disk 5 and 6 samples.

During data analysis it was observed that the scanned points on these disks did not match the design radius values well; the measured cylindrical radii were larger than the design values for every disk scanned. The differences in the design and actual sample radii were likely due to deformation of the sample from the release of residual stresses in the inner and outer windows after sampling. Therefore, to define an ideal surface against which to compare the scan points, a cylinder was fitted to the undamaged portion of the interior surface of each individual disk. The best-fit cylindrical radius was allowed to vary for each individual disk in order to provide the most accurate basis of comparison for measurement of erosion from the undamaged surface.

\section{RESULTS AND DISCUSSION}

A total of 28 target samples were scanned from ten consecutive targets: Targets 10-19. The raw scanning data results were analyzed to calculate metrics relevant to cavitation-induced erosion, which are shown in Table 1. 


\begin{tabular}{|c|c|c|c|c|c|c|c|c|c|}
\hline \multicolumn{2}{|c|}{ Sample } & \multicolumn{3}{|c|}{ Position of Maximum Erosion } & \multirow{2}{*}{$\begin{array}{c}\text { Maximum Erosion } \\
\text { Depth [in] }\end{array}$} & \multirow{2}{*}{$\begin{array}{c}\text { Remaining } \\
\text { Thickness [in] }\end{array}$} & \multirow{2}{*}{$\begin{array}{c}1 \mathrm{~cm} \text { Mean Depth } \\
\text { of Erosion* [in] }\end{array}$} & \multirow{2}{*}{$\begin{array}{c}2 \mathrm{~cm} \text { Mean Depth } \\
\text { of Erosion* [in] }\end{array}$} & \multirow{2}{*}{\begin{tabular}{|c|} 
Total Damaged \\
Area** $-\left[\mathrm{in}^{2}\right]$
\end{tabular}} \\
\hline Target & Disk & $\mathbf{x}$ & $\mathbf{y}$ & $\mathbf{z}$ & & & & & \\
\hline Target 10 & Disk 5 & N/A & N/A & N/A & 0.068 & 0.049 & N/A & N/A & N/A \\
\hline Target 11 & Disk 5 & N/A & N/A & N/A & 0.066 & 0.049 & N/A & N/A & N/A \\
\hline \multirow{3}{*}{ Target 12} & Disk 6 & N/A & N/A & N/A & 0.012 & 0.108 & N/A & N/A & N/A \\
\hline & Disk 13 & 0.336 & 0.097 & -2.049 & 0.121 & 0 & 0.033 & 0.019 & 3.031 \\
\hline & Disk 17 & 0.409 & 0.087 & -2.001 & 0.073 & 0.045 & 0.024 & 0.015 & 3.313 \\
\hline \multirow{3}{*}{ Target 13} & Disk 6 & N/A & N/A & N/A & 0.052 & 0.044 & N/A & N/A & N/A \\
\hline & Disk 13 & 0.653 & -0.069 & -2.045 & 0.132 & $0.005^{+}$ & 0.032 & 0.013 & 2.369 \\
\hline & Disk 17 & 0.590 & -0.018 & -2.017 & 0.096 & 0.035 & 0.016 & 0.009 & 2.496 \\
\hline \multirow{3}{*}{ Target 14} & Disk 6 & N/A & N/A & N/A & 0.036 & 0.062 & $\mathrm{~N} / \mathrm{A}$ & $\mathrm{N} / \mathrm{A}$ & $\mathrm{N} / \mathrm{A}$ \\
\hline & Disk 13 & 0.660 & 0.004 & -2.001 & 0.089 & 0.053 & 0.018 & 0.006 & 0.416 \\
\hline & Disk 17 & 0.625 & -0.061 & -2.005 & 0.089 & 0.047 & 0.019 & 0.007 & 0.371 \\
\hline \multirow{3}{*}{ Target 15} & Disk 6 & N/A & N/A & N/A & 0.006 & 0.091 & N/A & N/A & N/A \\
\hline & Disk 13 & 0.577 & -0.091 & -1.991 & 0.072 & 0.067 & 0.010 & 0.003 & 0.191 \\
\hline & Disk 17 & 0.530 & -0.224 & -1.960 & 0.047 & 0.088 & 0.006 & 0.002 & 0.155 \\
\hline \multirow{3}{*}{ Target 16} & Disk 6 & N/A & N/A & N/A & 0.008 & 0.084 & N/A & N/A & N/A \\
\hline & Disk 13 & 0.337 & 0.112 & -1.927 & 0.005 & 0.117 & 0.002 & 0.001 & 0.114 \\
\hline & Disk 17 & 0.482 & -0.188 & -1.922 & 0.008 & 0.116 & 0.003 & 0.002 & 0.383 \\
\hline \multirow{3}{*}{ Target 17} & Disk 6 & N/A & N/A & N/A & 0.022 & 0.072 & N/A & N/A & N/A \\
\hline & Disk 13 & 0.497 & -0.102 & -1.964 & 0.044 & 0.078 & 0.007 & 0.003 & 0.227 \\
\hline & Disk 17 & 0.406 & -0.200 & -1.960 & 0.042 & 0.077 & 0.011 & 0.006 & 0.353 \\
\hline \multirow{4}{*}{ Target 18} & Disk 5 & N/A & N/A & N/A & 0.120 & 0 & N/A & N/A & N/A \\
\hline & Disk 6 & N/A & N/A & N/A & 0.006 & 0.087 & N/A & N/A & N/A \\
\hline & Disk 13 & $\triangle$ & $\triangle$ & $\triangle$ & $\triangle \triangle$ & $\triangle \triangle$ & $\triangle$ & $\triangle$ & $\triangle$ \\
\hline & Disk 17 & $\triangle$ & $\triangle$ & $\triangle$ & $\triangle \triangle$ & $\triangle \triangle$ & $\triangle$ & $\triangle$ & $\triangle$ \\
\hline \multirow{4}{*}{ Target 19} & Disk 5 & N/A & N/A & N/A & 0.120 & 0 & N/A & N/A & N/A \\
\hline & Disk 6 & N/A & N/A & N/A & 0.004 & 0.087 & N/A & N/A & N/A \\
\hline & Disk 13 & $\triangle$ & $\triangle$ & $\triangle$ & 0.006 & 0.190 & $\triangle$ & $\triangle$ & $\triangle$ \\
\hline & Disk 17 & $\triangle$ & $\triangle$ & $\triangle$ & 0.004 & 0.190 & $\triangle$ & $\triangle$ & $\triangle$ \\
\hline \multicolumn{10}{|c|}{ * Mean depth of erosion circles were centered on deepest erosion point measured } \\
\hline \multicolumn{10}{|c|}{ ** "Damaged Area" defined by depth deviation of $>0.0015$ inch from ideal model surface, after trimming points from regions showing no damage in photos } \\
\hline \multicolumn{10}{|c|}{ + Disk 13 from Target 13 contained the leak location and remaining thickness is zero at the hole, 0.005 in was an artifact from the scanner resolution } \\
\hline \multicolumn{10}{|c|}{$\triangle$ Pits were small and scattered; no single point of appreciable maximum erosion was present } \\
\hline
\end{tabular}

Table 1. Calculated data from laser line scans of samples from Targets 10-19.

\subsection{DISK 13 AND 17 SAMPLES}

The importance of laser line scanning characterization of erosion damage was realized after the Target 12 and 13 leaks, and as a result the Disk 13 and 17 locations have been the most scrutinized locations in the SNS mercury target vessel. The Disk 13/17 locations were scanned for eight SNS targets, two that leaked during service and six that did not leak, and the results revealed insight into the depth and location of maximum erosion for these samples.

The distribution of the material eroded from Disk 13 from Target 12 (Disk 13/Target 12) and Disk 17 from Target 12 (Disk 17/Target 12), are shown in Figure 20. The damage to these disks had two general types of erosion damage present: (1) relatively shallow ( $<0.010$ in) dimple-like pitting and (2) deep cavitylike erosion ( $>0.050 \mathrm{in})$. The shallow pitting-type erosion is shown in the plots of the material eroded are in the blue to purple range, which corresponds to an erosion depth of approximately 0 to -0.020 in and is found on the majority of the sample surface. The areas with deep cavity-type erosion are shown in the erosion plots as the green to red range, and are located near the center of the sample. The leak was located 
in Disk 13 and the maximum depth of erosion was 0.121 in, which completely perforated the sample at the leak location. Maximum erosion to Disk 17/Target 12 was 0.073 in, and 0.045 in of thickness remained at this location.

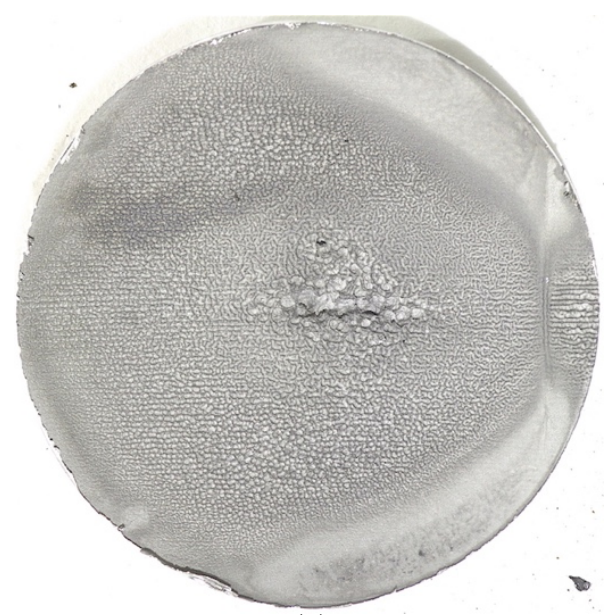

(a)

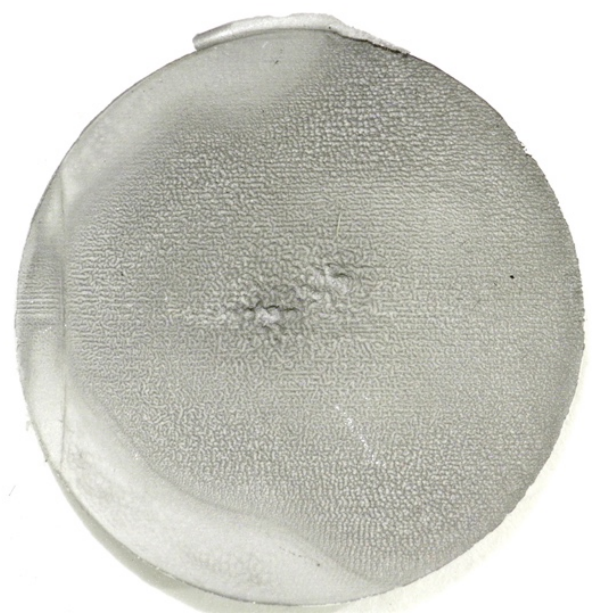

(c)

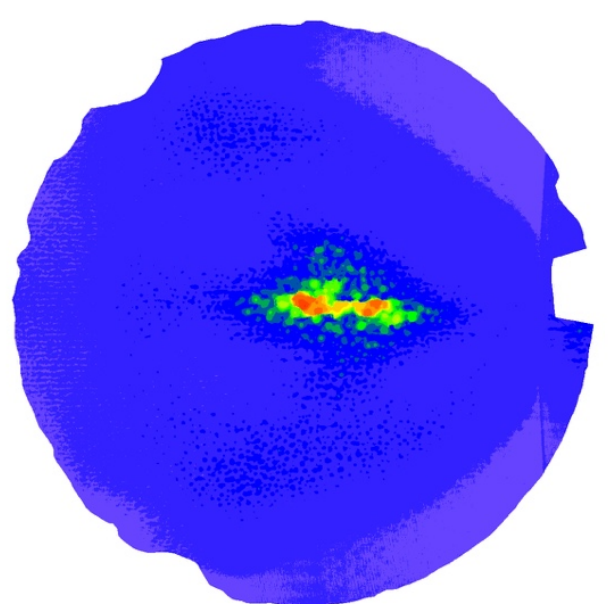

Material Removed [in]

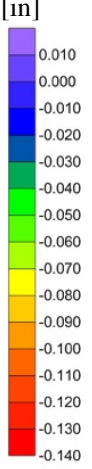

(b)

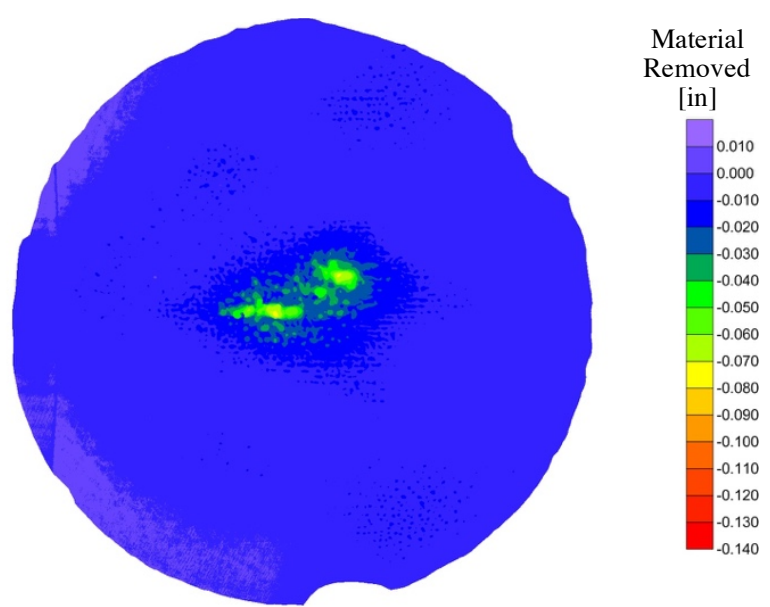

(d)

Figure 20. (a) Image and (b) distribution of the material eroded from Disk 13/Target 12, which contained the Target 12 leak, and (c) image and (d) distribution of material eroded from Disk 17/Target 12.

Images and distribution of material removed for the Disk 13 and 17 locations in Target 13 are shown in Figure 21. The erosion damage morphology on the Target 13 Disks $13 / 17$ were very similar to that observed on the samples from Target 12. That is, small cavities of deep erosion near the center of the disks surrounded by shallow dimple-like pitting. Target 13 also leaked at the Disk 13 location, and both targets had more pronounced cavity-type erosion on the driver side of the target. The deepest erosion measured on Disk 17/Target 13 was 0.096 in with 0.035 in of material remaining in the thickness, which was slightly deeper than the deepest cavity measured on Disk 17/Target 12. 


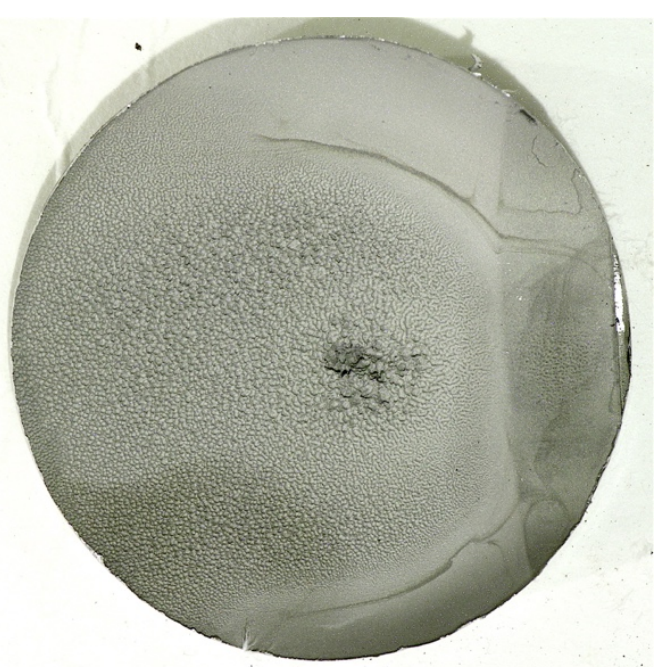

(a)

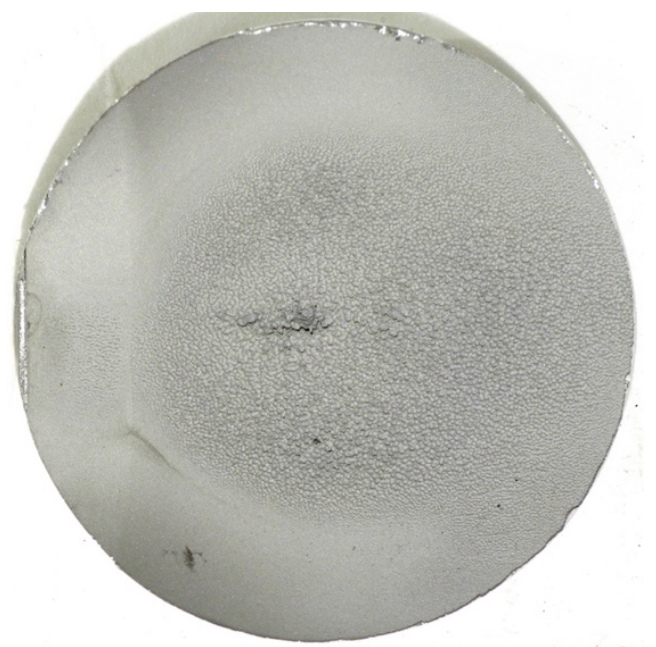

(c)

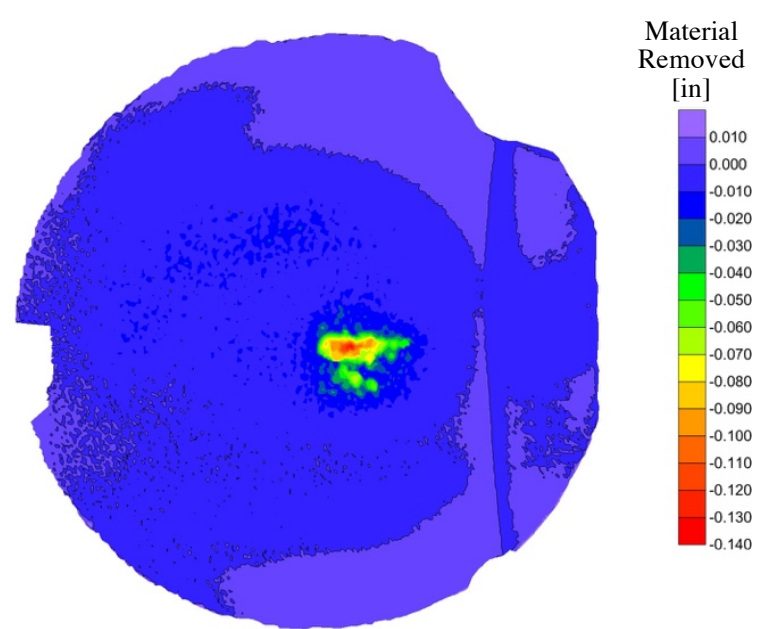

(b)

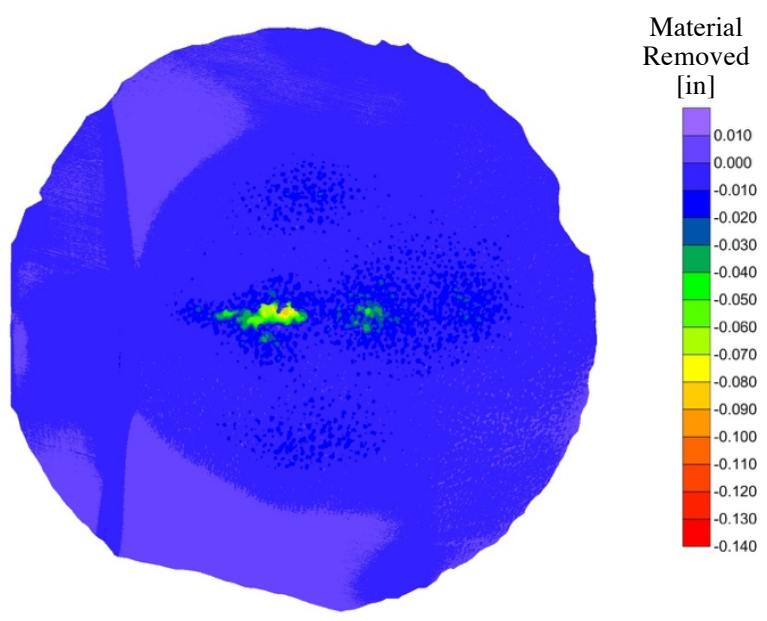

(d)

Figure 21. (a) Image and (b) distribution of the material eroded from Disk 13/Target 13, which contained the Target 13 leak, and (c) image and (d) distribution of material eroded from Disk 17/Target 13.

The target management plan was put into place after the leaks in Targets 12 and 13, therefore Target 14 and 15 were the first original design targets operated at fixed powers for a prescribed time period. Erosion patterns on the Disks 13 and 17 samples from Targets 14 and 15 were similar to those observed on Targets 12 and 13, but had a more "comet tail" appearance around the deep-erosion cavities, as shown in Figure 22. It is possible that the same pattern was present on the Target 12 and 13 samples during the early phase of operation, and the dimple-type erosion on around the "comet tail" cavity-type erosion appeared at a later point during operation. The maximum erosion depth was 0.089 in for both Disk 13 and 17 from Target 14, while the maximum erosion depth measured in Target 15 was 0.072 in on Disk 13. 

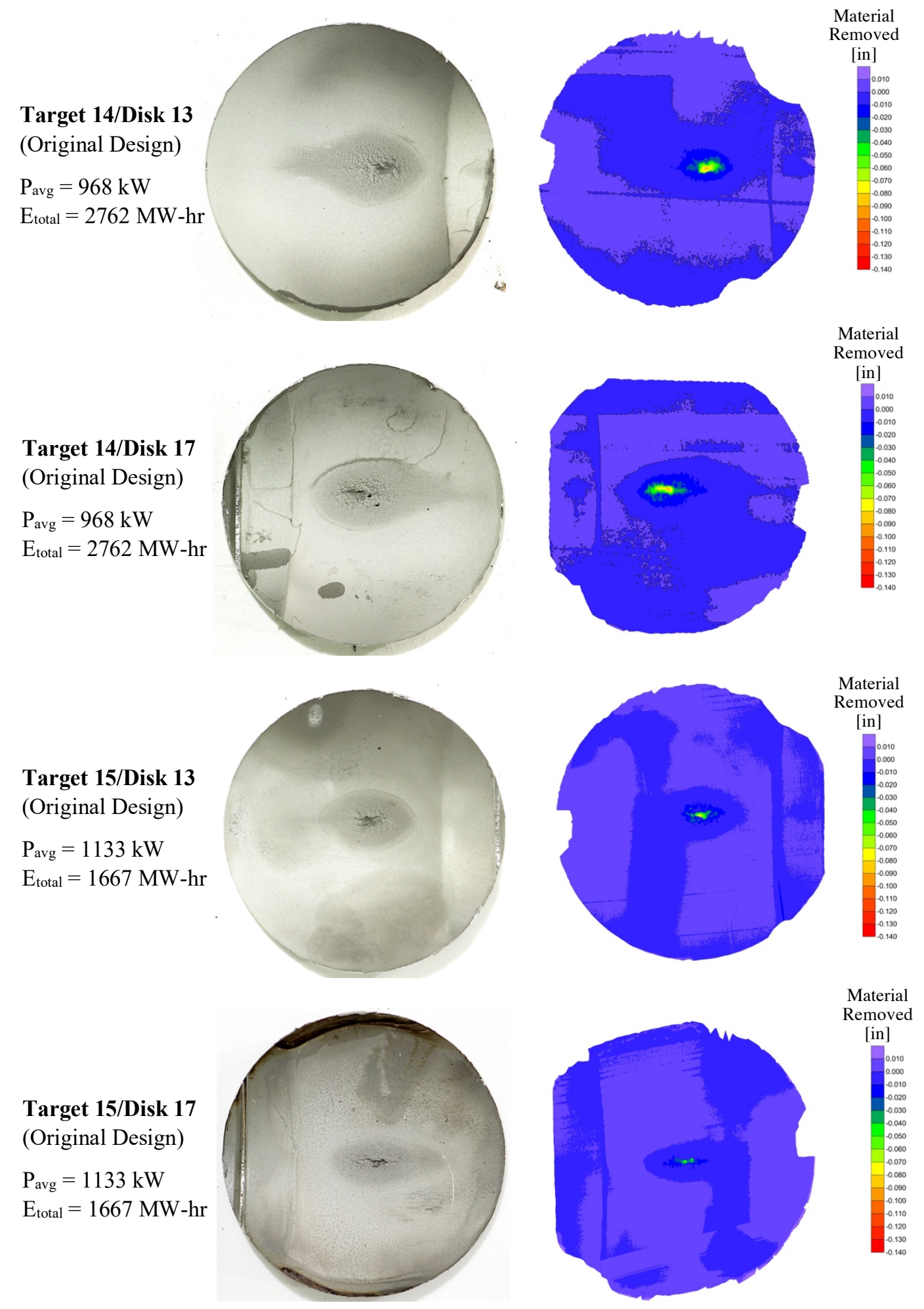

Figure 22. Images and erosion plots for Disks 13 and 17 from Targets 14 and 15 (original design). 
Scans of Disks 13 and 17 from Targets 16 and 17 were the first erosion measurements of samples from the end of supply passages of a jet-flow design target. The images and plots of the material removed from the Target 16 and 17 samples are shown in Figure 23.

\section{Target 16/Disk 13 (Jet-flow Design) \\ $\mathrm{P}_{\text {avg }}=968 \mathrm{~kW}$ \\ $\mathrm{E}_{\text {total }}=1780 \mathrm{MW}-\mathrm{hr}$}

\section{Target 16/Disk 17}

(Jet-flow Design)

$\mathrm{P}_{\text {avg }}=968 \mathrm{~kW}$

$\mathrm{E}_{\text {total }}=1780 \mathrm{MW}-\mathrm{hr}$

\section{Target 17/Disk 13} (Jet-flow Design)

$\mathrm{P}_{\mathrm{avg}}=1127 \mathrm{~kW}$

$\mathrm{E}_{\text {total }}=1936 \mathrm{MW}-\mathrm{hr}$
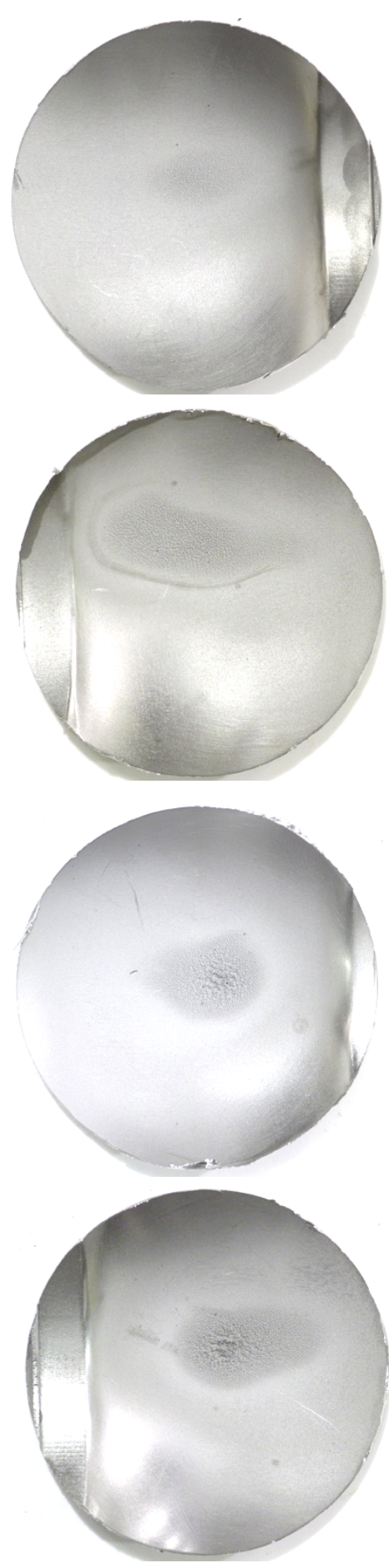

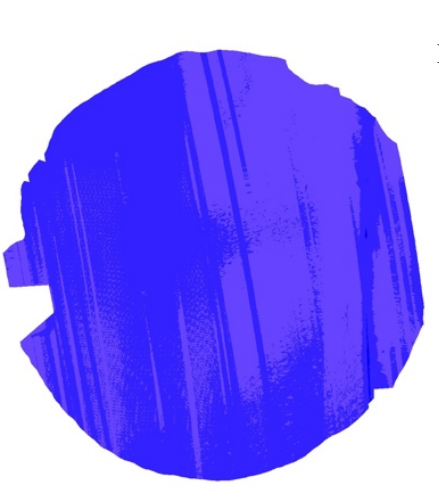

Material moved [in]

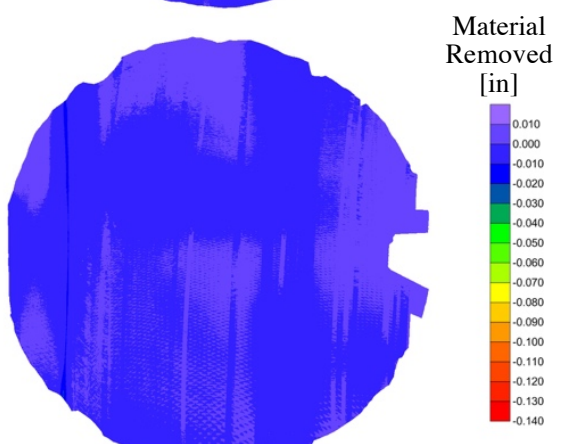

Material Removed

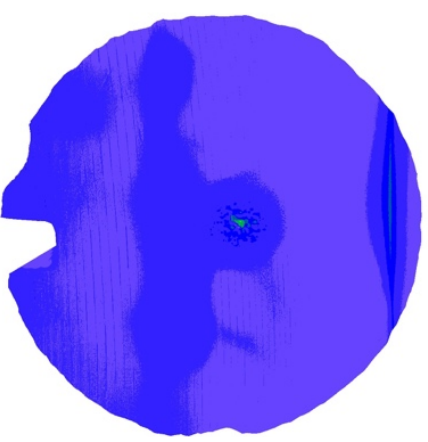

[in]

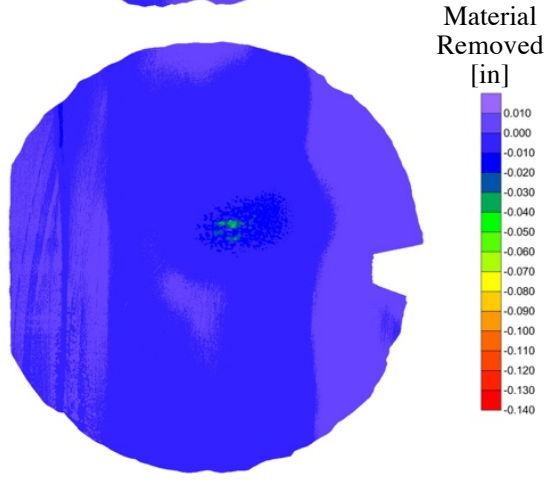

Target 17/Disk 17 (Jet-flow Design)

$\mathrm{P}_{\text {avg }}=1127 \mathrm{~kW}$

$\mathrm{E}_{\text {total }}=1936 \mathrm{MW}-\mathrm{hr}$

Figure 23. Images and erosion plots for Disks 13 and 17 from Targets 16 and 17 (jet-flow design). 
While the "comet tail" shapes were present on Disks 13 and 17 from Targets 16 and 17, the erosion damage to the end of the supply passages was less pronounced in these two jet-flow targets. The maximum depth of erosion measured for Target 16 Disks 13 and 17 were 0.005 and 0.008 in, respectively. Whereas, the maximum depth of erosion measured for Target 17 Disk 13 and 17 were 0.044 and 0.042 in, respectively. Target 17 was operated at an average power of $1127 \mathrm{~kW}$, which was approximately $16 \%$ higher than the average power of Target $16(968 \mathrm{~kW})$ and might account for the increase erosion measured in Target 17.

Targets 18 and 19 were the first two SNS targets to operate with small-bubble gas injection, which reduced the erosion damage to the inner wall, as shown earlier in Figure 12. Images of the Disk 13 and 17 samples from these two targets showed some areas with a dull-finish discoloration, areas where the surface finish was not shiny, but no obvious regions of erosion were observed. The laser scan results verified that very little erosion damage occurred to the Disk 13 and 17 specimens from Targets 18 and 19. Images and plots of the material removed from Disks 13 and 17 removed from Targets 18 and 19 are shown in Figure 24.

While several areas on the images appear to have patches of erosion, the scanning results show that these features were within the resolution of the scanner $(\leq 0.001$ in). The lack of material removal from the surface of the Target 18 and 19 samples complicated the data analysis and produced artifacts on the plots of the material removed. For example, the vertical green colored "bars" observed on the plot of material removed from Target 18 were a result of the data analysis where a transition between the spherical geometric primitives occurred. Other patterns on the material removal plots for the Target 18 and 19 specimens might have been generated by the release of residual stresses built up in the target vessel during fabrication, which distort the surfaces from the as-designed.

Some of the patterns of discoloration with a dull finish on the samples from Targets 18 and 19 appeared to be associated with pits on the sample surfaces. Close examination of the images and scan results show that several of the isolated pits were located at the leading edge of the patterns, as shown in Figure 25. The regions with a dull or discolored finish appeared to emanate from the isolated pits, similar to a comet head and tail configuration. However the orientation of the comet head/tail appears opposite to the direction of the bulk mercury flow. That is, the bulk mercury flow direction flows from the tail to the head of the discolored areas on the Disk 13 and 17 samples from Targets 17 and 18. It is not clear why the "tails" of the discolored features appear opposite of the flow direction, but it is possible that a layer of recirculated mercury against the target wall flowed in the direction opposite to the bulk mercury flow. More detailed computational fluid dynamics analysis of the flow behavior against the vessel wall at the end of the supply passages are ongoing to better understand the erosion damage observations and mercury flow behavior in these areas. 
Target 18/Disk 13 (Jet-flow Design)

$\mathrm{Q}_{\text {avg }}=0.25-0.45 \mathrm{SLPM}$

$\mathrm{P}_{\mathrm{avg}}=1128 \mathrm{~kW}$

$\mathrm{E}_{\text {total }}=1261 \mathrm{MW}-\mathrm{hr}$

Target 18/Disk 17

(Jet-flow Design)

$\mathrm{Q}_{\mathrm{avg}}=0.25-0.45 \mathrm{SLPM}$

$\mathrm{P}_{\text {avg }}=1128 \mathrm{~kW}$

$\mathrm{E}_{\text {total }}=1261 \mathrm{MW}-\mathrm{hr}$

\section{Target 19/Disk 13}

(Jet-flow Design)

$\mathrm{Q}_{\mathrm{avg}}=\sim 0.45 \mathrm{SLPM}$

$\mathrm{P}_{\mathrm{avg}}=1207 \mathrm{~kW}$

$\mathrm{E}_{\text {total }}=1987 \mathrm{MW}-\mathrm{hr}$

Target 19/Disk 17

(Jet-flow Design)

$\mathrm{Q}_{\mathrm{avg}}=\sim 0.45 \mathrm{SLPM}$

$\mathrm{P}_{\mathrm{avg}}=1207 \mathrm{~kW}$

$\mathrm{E}_{\text {total }}=1987 \mathrm{MW}-\mathrm{hr}$
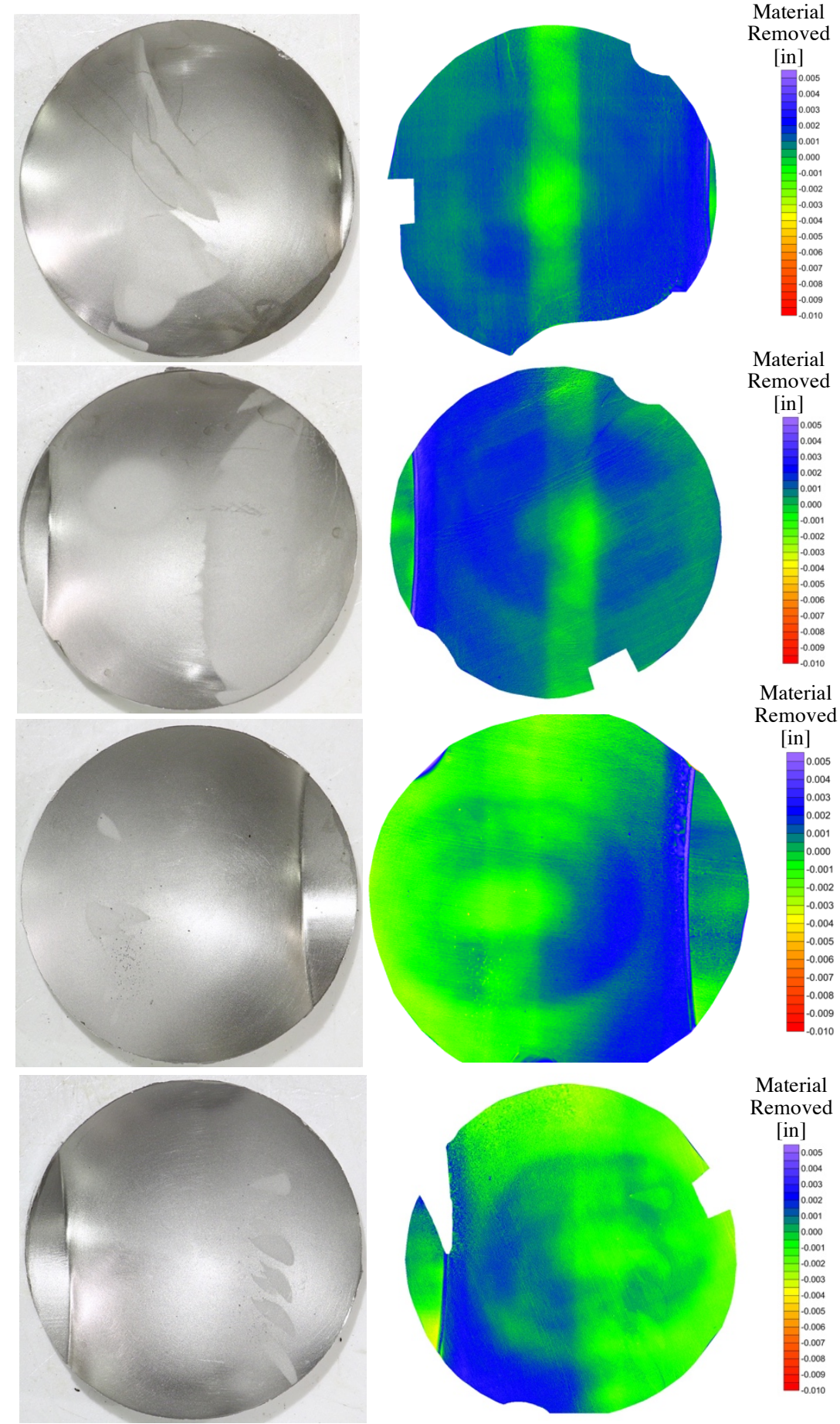

moved

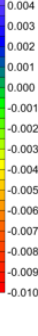

Material

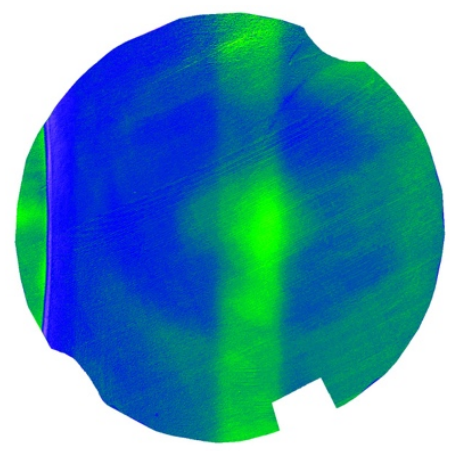

[in]

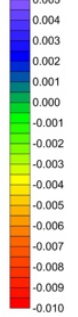

Material

Removed

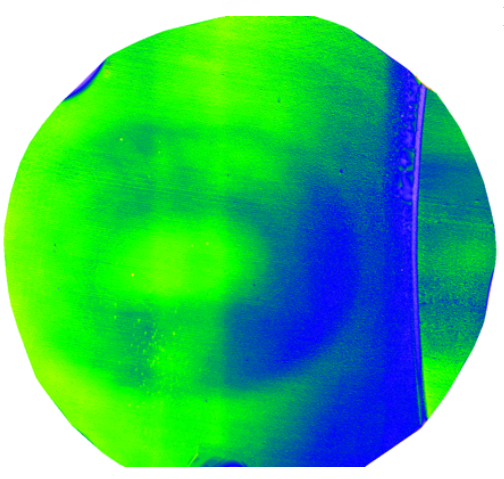

[in]

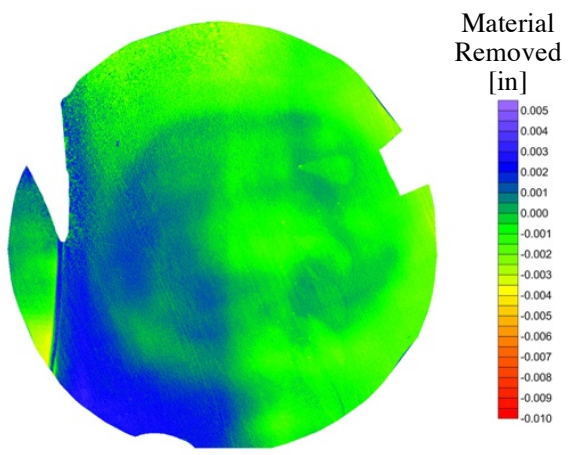

Figure 24. Images and erosion plots for Disks 13 and 17 from Targets 18 and 19 (jet-flow design with gas injection). 

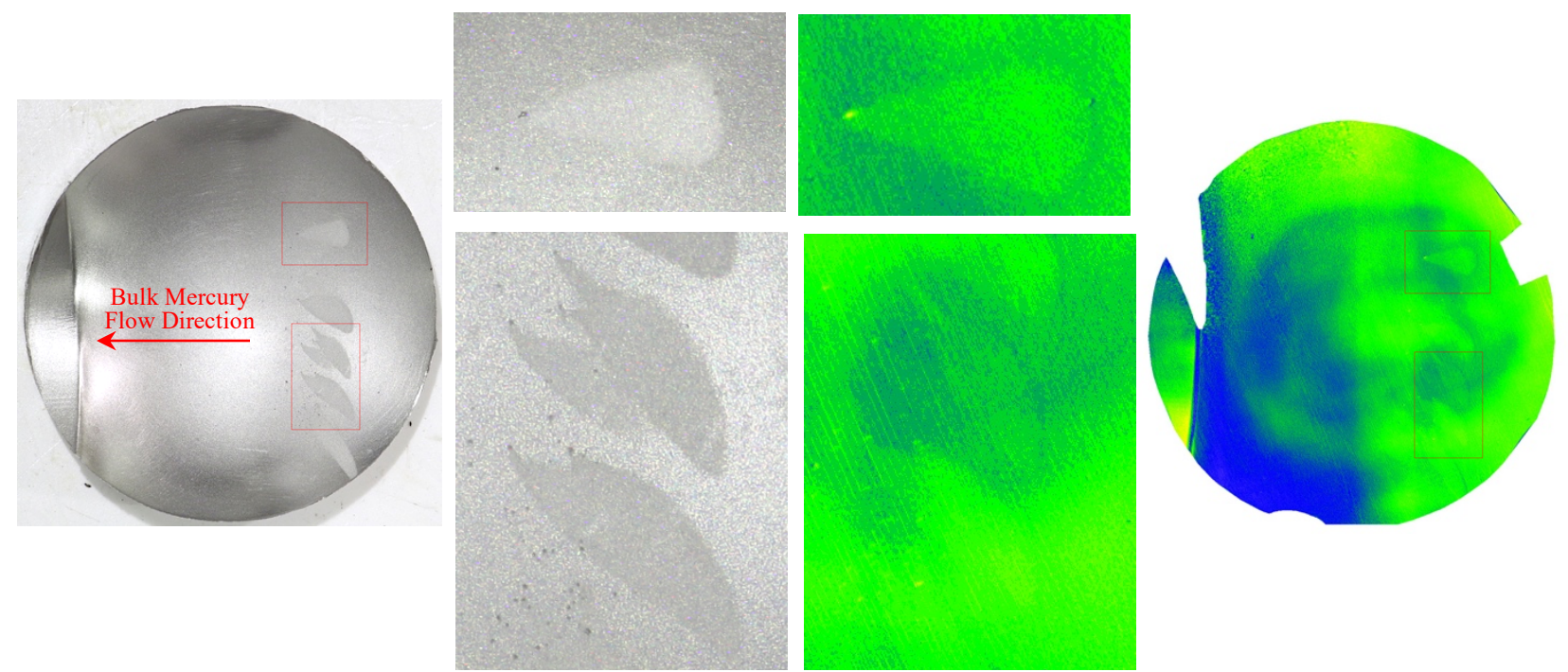

Figure 25. Images and erosion plots for Disks 17 Target 19 showing magnified views of regions containing "pit/comet" discolored features.

The location where the maximum depth of erosion occurred was calculated from the scan data for each Disk 13/17. The location of maximum depth of erosion for the Disk 13/17 samples scanned for all targets is overlaid together on one figure in Figure 26; locations from the Disk 17 samples are mirrored onto the locations for the Disk 13 samples. The location of the maximum erosion was similar for each Disk 13/17 sample; all the points of maximum erosion were contained within a minimum bounding circle with a 0.370 in diameter.

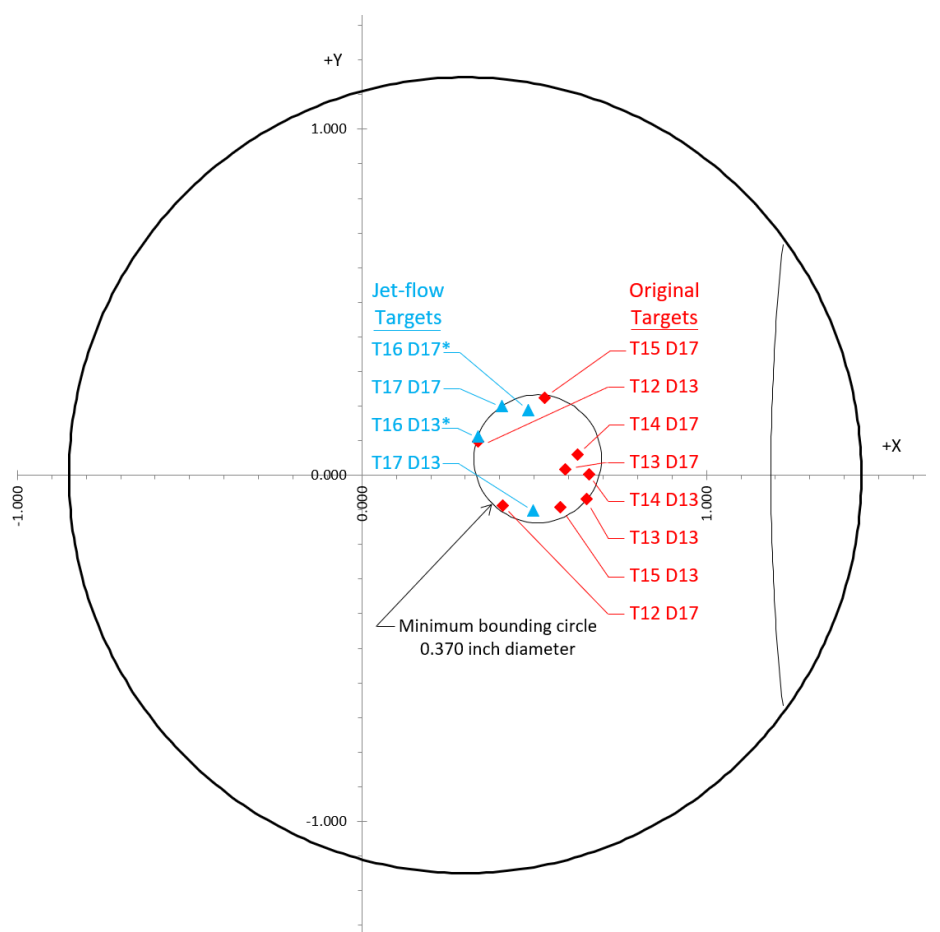

Figure 26. Locations of the maximum depth of erosion for the Disk 13 and 17 samples overlaid onto one figure; locations for Disk 17 samples are mirrored onto the Disk 13 locations. (*Both disks from Target 16 showed shallow erosion with no single, well-defined, deep point. The point of maximum erosion was defined to be the center of a wider-than-normal area of deep points found.) 


\subsection{DiSK 5 SAMPLES}

The samples from the center of the mercury vessel inner wall at the beam entrance window (Disk 5) experience the greatest erosion damage during operation, and are often fractured along the window's horizontal centerline, as shown earlier in Figure 5. Currently there is no method available to hold half-disk sections during laser scans, and half-disk samples were not scanned. Of the target samples scanned to date, only four targets had Disk 5 samples that were not fractured, and these specimens were scanned to produce material removal plots. The erosion data reported in Table 1 and shown in the material removal plots for Disk 5 samples were for material eroded from the sample surface facing the bulk mercury flow. Erosion to the window-flow facing surfaces were primarily shallow "comet tail" type of erosion pits, which did not progress as extensively as the damage to the bulk-flow facing surfaces.

Target 10 was the first jet-flow target design operated at the SNS, but was operated to only 601 MW-hr due to a leak that developed at a weld joining the target front body and transition sections. The short life of Target 10 provided an opportunity to obtain information on the progress of erosion damage during the early stages of operation. Also, the short life of Target 6 provided an almost direct comparison of the evolution of damage between original and jet-flow target designs, which was shown in Figure 7. The erosion patches on the Target 10 Disk 5 sample were not centered and had a morphology the was oriented parallel to the direction of the jet flow against the inner wall. A photograph and erosion plots of the Disk 5 sample from Target 10 are shown in Figure 27. The erosion plots are on two different length scales to fully illustrate details of the erosion patterns. Three large areas of erosion were present: one large patch at approximately the eight o'clock position on the disk, and two areas just above the horizontal center line. The maximum depth of erosion was 0.068 in and occurred in the patch above the centerline on the left. This pattern has not been observed on other SNS target inner windows and it is not clear if flow-induced erosion might have contributed to the erosion damage observed on the Target 10 inner window.
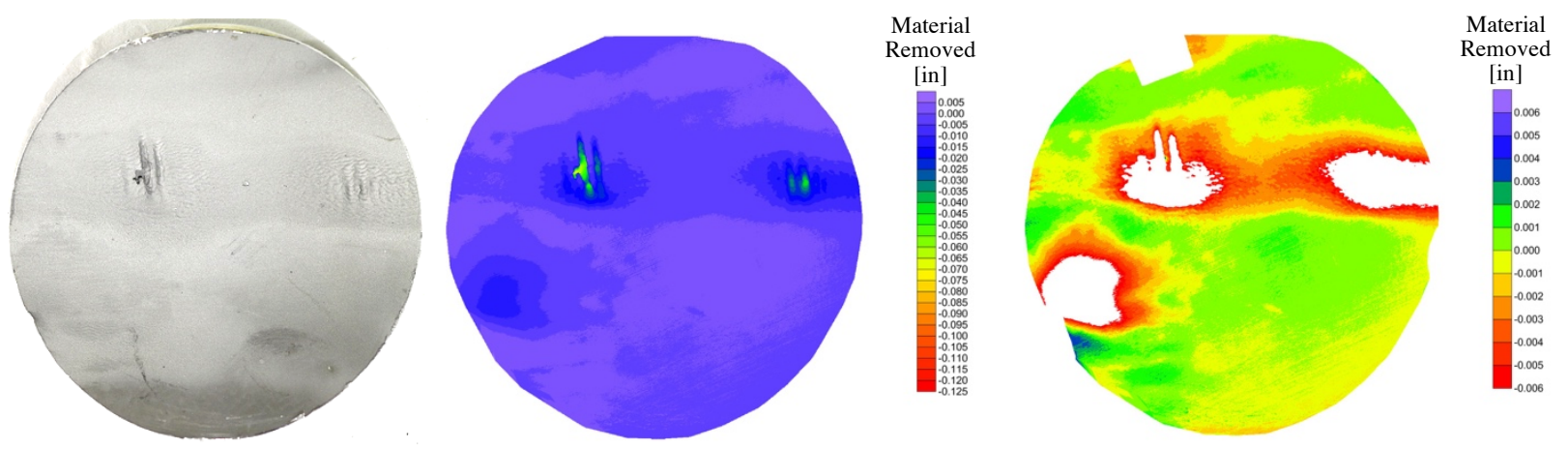

Figure 27. Image and erosion plots for Disk 5 from Target 10 (jet-flow design).

Target 11 developed a leak early during operation and, to date, is the shortest-operated SNS target, operating for approximately $166 \mathrm{MW}-\mathrm{hr}$ before leaking. While the Target 11 leak interrupted neutron production, it also provided information on the evolution of cavitation-induced erosion damage to the inner wall of an original target design. Erosion damage to the Target 11 inner wall consisted of a band of damage along the horizontal centerline of the sample with small erosion patches above and below the disk center, as shown in Figure 28. Note - the dark circular patterns on the left-hand side of the Target 11/Disk 5 photograph in Figure 28 are stains from the solutions used to clean the sample, which is confirmed by the fact that these discolored features do not appear in the laser scanning data. The erosion pattern at the disk center is consistent with the early stages of the $\Phi$-shaped damage pattern development, which have been 
observed in original design targets operated to higher total exposure levels, as shown in Figures 5 and 7. The damage pattern at the center of the sample might be a contributing factor to the fracture of the inner wall by providing a stress concentration along the horizontal centerline of the wall, which might increase the local stresses and initiate fracture during fatigue loading of the inner wall.
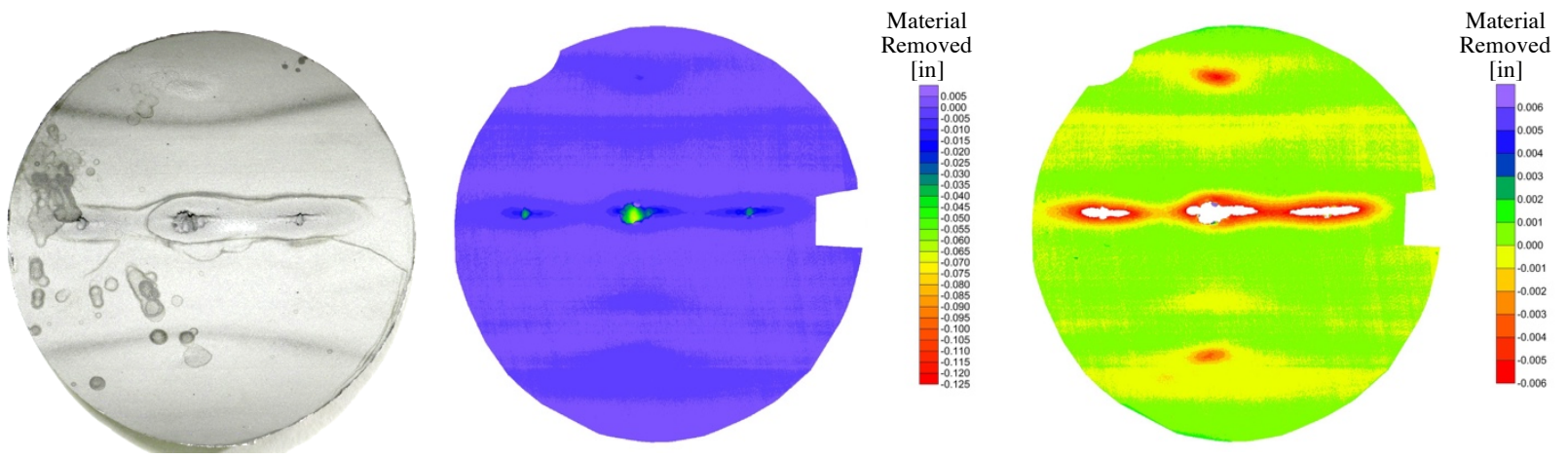

Figure 28. Image and erosion plots for Disk 5 from Target 11 (original design).

After Target 11, the mercury vessel inner wall was fractured on all subsequent targets until gas injection was implemented in Target 18, which did not have a fractured inner wall. Target 18 was operated with a gas injection flow rate of $0.25-0.45$ SLPM and at an average power of $1.1 \mathrm{MW}$ to a total absorbed energy of $1261 \mathrm{MW}-\mathrm{hr}$. A photograph and erosion maps for the Disk 5 sample from Target 18 are shown in Figure 29. Similar to the erosion patterns on Target 10, the erosion damage at the center of the Target 18/Disk 5 had a morphology oriented parallel to the direction of the jet flow against the inner wall. However, unlike the center sample from Target 10, the damage to the Target 18/Disk 5 was located at the center of the sample and perforated the sample at four locations. Another small patch of erosion was located above the damage in the center of the disk, on the downstream side of the jet-flow against the inner wall.
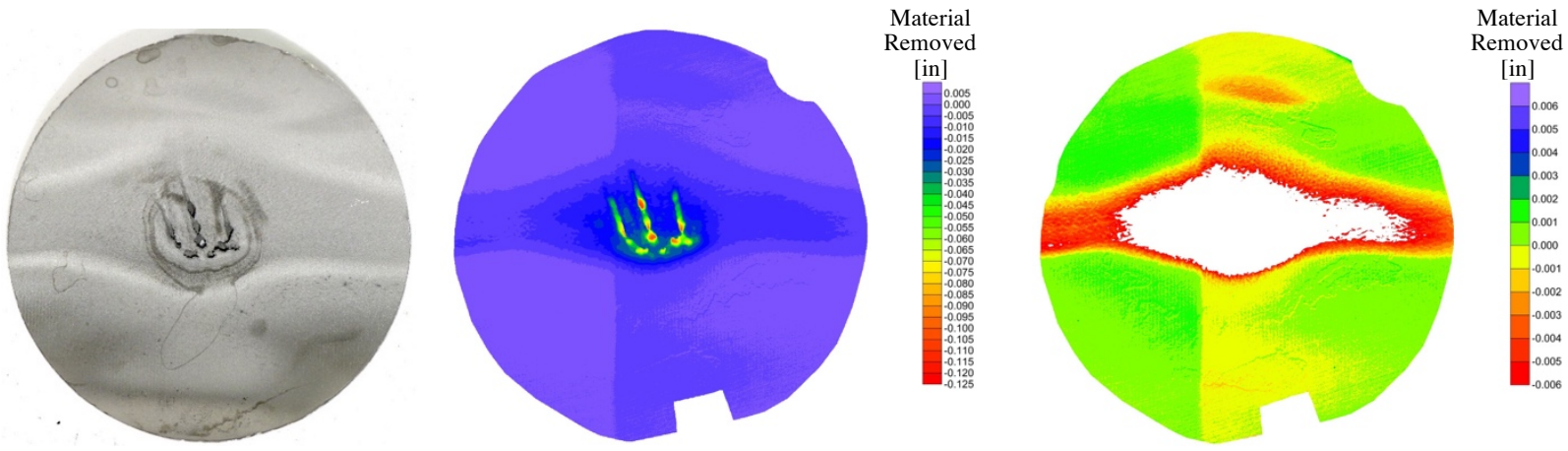

Figure 29. Image and erosion plots for Disk 5 from Target 18 (jet-flow design with gas injection).

Target 19 was the second target operated with gas injection, which operated with a gas injection rate of approximately 0.45 SLPM at an average power of $\sim 1.2 \mathrm{MW}$ to a total energy of $1987 \mathrm{MW}$-hr. Similar to Target 18, the erosion damage to the Target 19 inner wall was reduced relative to other high-use targets operated without gas injection and the inner wall was not fractured. A photograph and erosion plots of the Disk 5 sample from Target 19 are shown in Figure 30. Similar to the center sample from the Target 18 inner wall, the erosion damage at the center of the Target 19 inner wall was concentrated in erosion "channels" oriented parallel to the direction of the jet flow against the inner wall. Also similar to Target 18, the sample from the center of Target 19 was perforated at four locations. Similar to the erosion morphology 
observed in jet-flow design Targets 10 and 18, it is possible the jet flow against the inner wall may have promoted flow-induced erosion that affected the erosion morphology observed in jet-flow design targets.
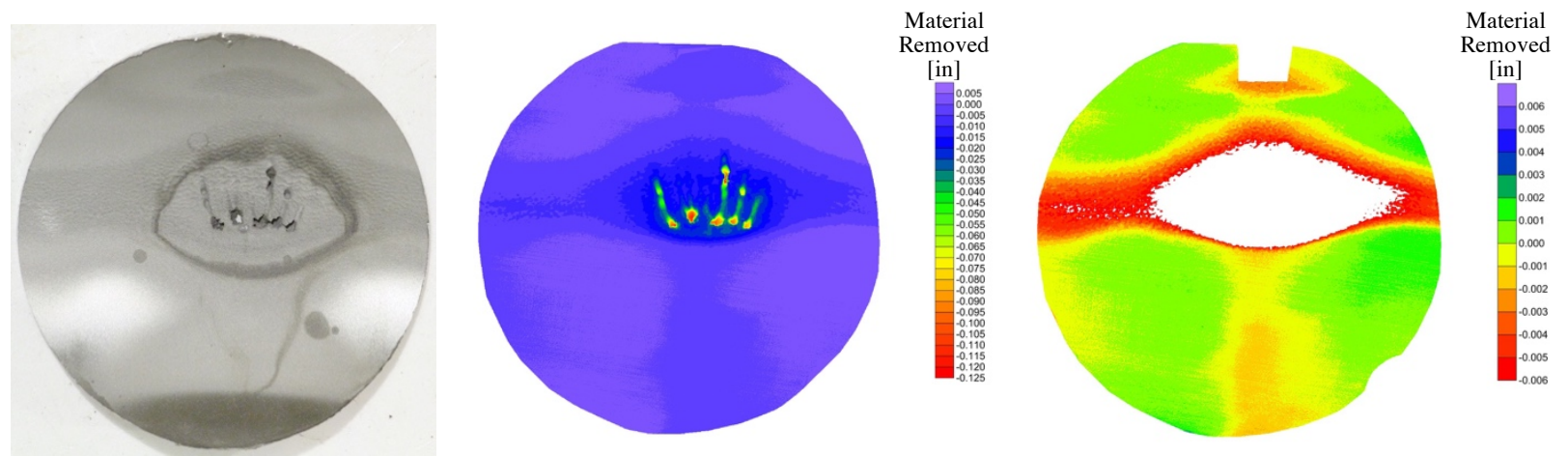

Figure 30. Image and erosion plots for Disk 5 from Target 19 (jet-flow design with gas injection).

\subsection{DISK 6 SAMPLES}

While the most severe erosion damage in SNS targets occurs on the mercury vessel inner wall, this layer of the target is not a leak boundary. Any perforation through the inner wall allows mercury to communicate between the bulk- and window-mercury flows, but does not allow mercury to enter the interstitial region between the shroud and mercury vessel. The leak boundary for the mercury vessel at the center of the beam entrance region is the outer wall. The samples from the center of the mercury vessel outer wall are designated Disk 6, and to date these samples have been scanned for eight target vessels.

Disk 6 samples from two original design SNS targets were scanned: Targets 12 and 13 . Photographs and erosion plots for the Disk 6 specimens from Targets 12 and 13 are shown in Figure 31. Both Target 12 and 13 were operated at approximately 964 and $1075 \mathrm{~kW}$ to 4460 and $2589 \mathrm{MW}-\mathrm{hr}$, respectively. The direction of the mercury flow is from bottom to top against the surfaces shown in Figure 31. The erosion damage to the window-facing surfaces of these Disk 6 samples was distinguished by relatively large round-shaped pits and smaller elongated pits that were somewhat randomly distributed on the samples. The maximum depths of erosion for the Disk 6 samples from Targets 12 and 13 were 0.012 and 0.052 in, respectively. On both samples a series of round relatively large pits were aligned horizontally just above the center of the disk, which were more pronounced on the Target 13 Disk 6 sample. A few small isolated features on the Target 12 Disk 6 sample appeared purple, indicating material above the disk surface, were attributed to swarf chips adhering to the sample during the scanning characterization. 
Target 12/Disk 6

(Original Design)

$\mathrm{P}_{\text {avg }}=964 \mathrm{~kW}$

$\mathrm{E}_{\text {total }}=4460 \mathrm{MW}-\mathrm{hr}$
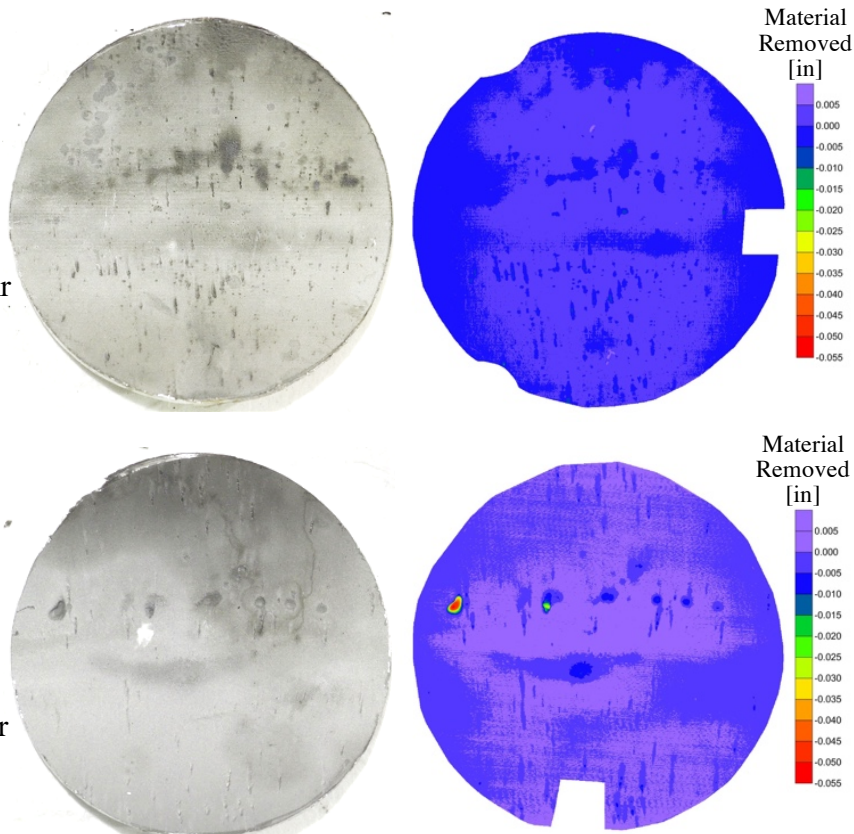
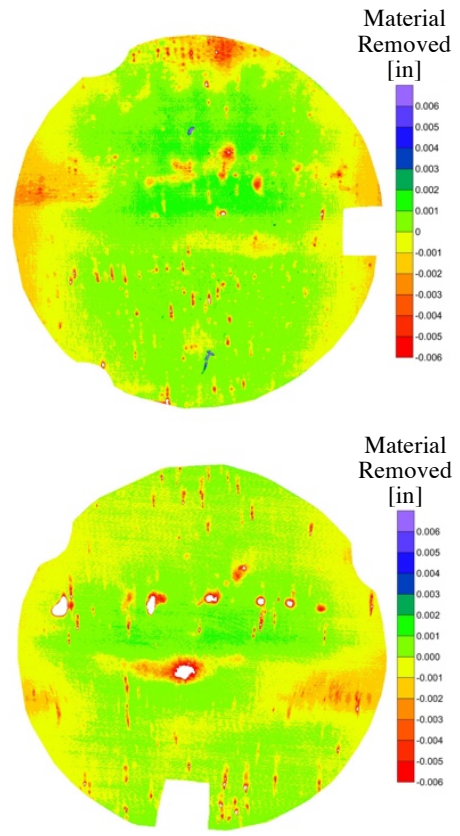

Figure 31. Images and erosion plots for Disk 6 samples from Targets 12 and 13 (original design without gas injection).

A total of four Disk 6 samples were scanned from jet-flow target designs: two were from jet-flow targets without gas injection and two were from jet-flow targets with gas injection. Targets 16 and 17 were jet-flow targets operated without gas injection at 965 and $11237 \mathrm{~kW}$ to 1780 and $1936 \mathrm{MW}-\mathrm{hr}$, respectively. Photographs and erosion plots for the Disk 6 samples from Targets 16 and 17 are shown in Figure 32. The maximum depth of erosion for the Disk 6 samples from Targets 16 and 17 were 0.008 and 0.022 in, respectively.

Target 16/Disk 6 (Jet-flow Design) No Gas Injection

$\mathrm{P}_{\mathrm{avg}}=968 \mathrm{~kW}$ $\mathrm{E}_{\text {total }}=1780 \mathrm{MW}-\mathrm{hr}$
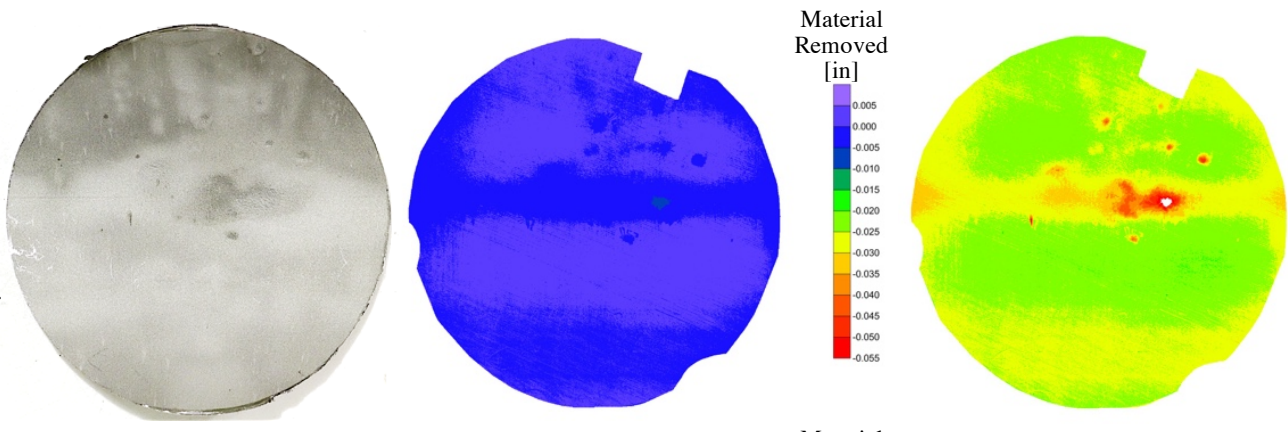

Material Removed

[in]
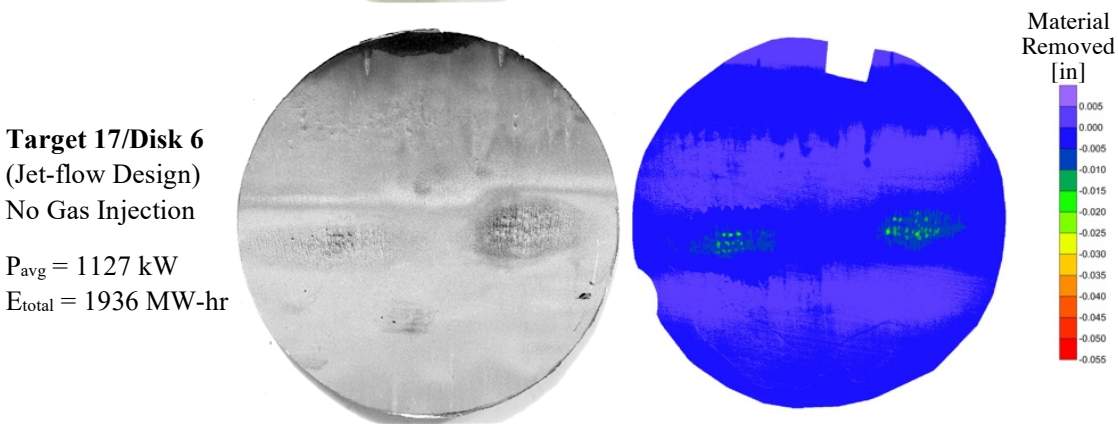

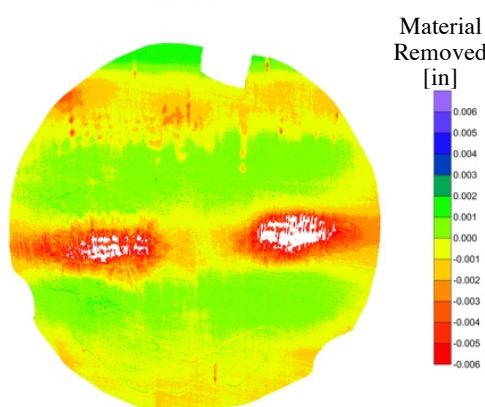

Figure 32. Images and erosion plots for Disk 6 samples from Targets 16 and 17 (jet-flow design without gas injection). 
The Disk 6 sample from Target 16 has a large area of erosion near the center of the disk and several relatively round large pits above the center region, with no large pits below the horizontal centerline of the sample. The erosion damage on the Disk 6 sample from Target 17 was different, with two large patches of erosion just below the sample horizontal center line, offset on either side of the vertical centerline. The Disk 6 from Target 17 also had a banded area of erosion damage toward the top of the sample characterized by both round and elongated pitting damage. Although Target 17 was operated at a higher power for a longer period of time compared to Target 16, it is not clear why the erosion patterns between the Disk 6 samples from Targets 16 and 17 are not similar. It is possible the pattern observed on the Target 16 sample was a precursor to the damage observed on Target 17, and additional operation of Target 16 might have yielded a similar "two patch" erosion pattern observed on Target 17.

Targets 18 and 19 were jet-flow design targets operated with gas injection at 1128 and $1207 \mathrm{~kW}$ to 1261 and 1987 MW-hr, respectively. The gas injection rates for Targets 18 and 19 were $0.25-0.45$ and 0.45 SLPM, respectively. The images and erosion plots for the Disk 6 samples from Targets 18 and 19 are shown in Figure 33. A similar erosion pattern was observed on the images of the Disk 6 samples from Targets 18 and 19, with a circular patch of erosion at the center of the samples and elongated patches of discoloration on either side of the sample center that were horizontally oriented. The maximum depth of erosion for the Disk 6 samples from Targets 18 and 19 were 0.006 and 0.004 in, respectively, and occurred in the center erosion patch on both samples. Also, vertically oriented bands of discoloration were observed on either side of the samples center, which were fainter on the Target 18 Disk 6 sample compared to the Target 19 Disk 6. While these patches of discoloration were obvious on the images of the samples, the laser scans did not indicate any erosion corresponding with these discolored areas. It is possible that the discoloration was caused by erosion to the sample surface that affected the reflectively of the surface but was shallower than the resolution of the scanner.

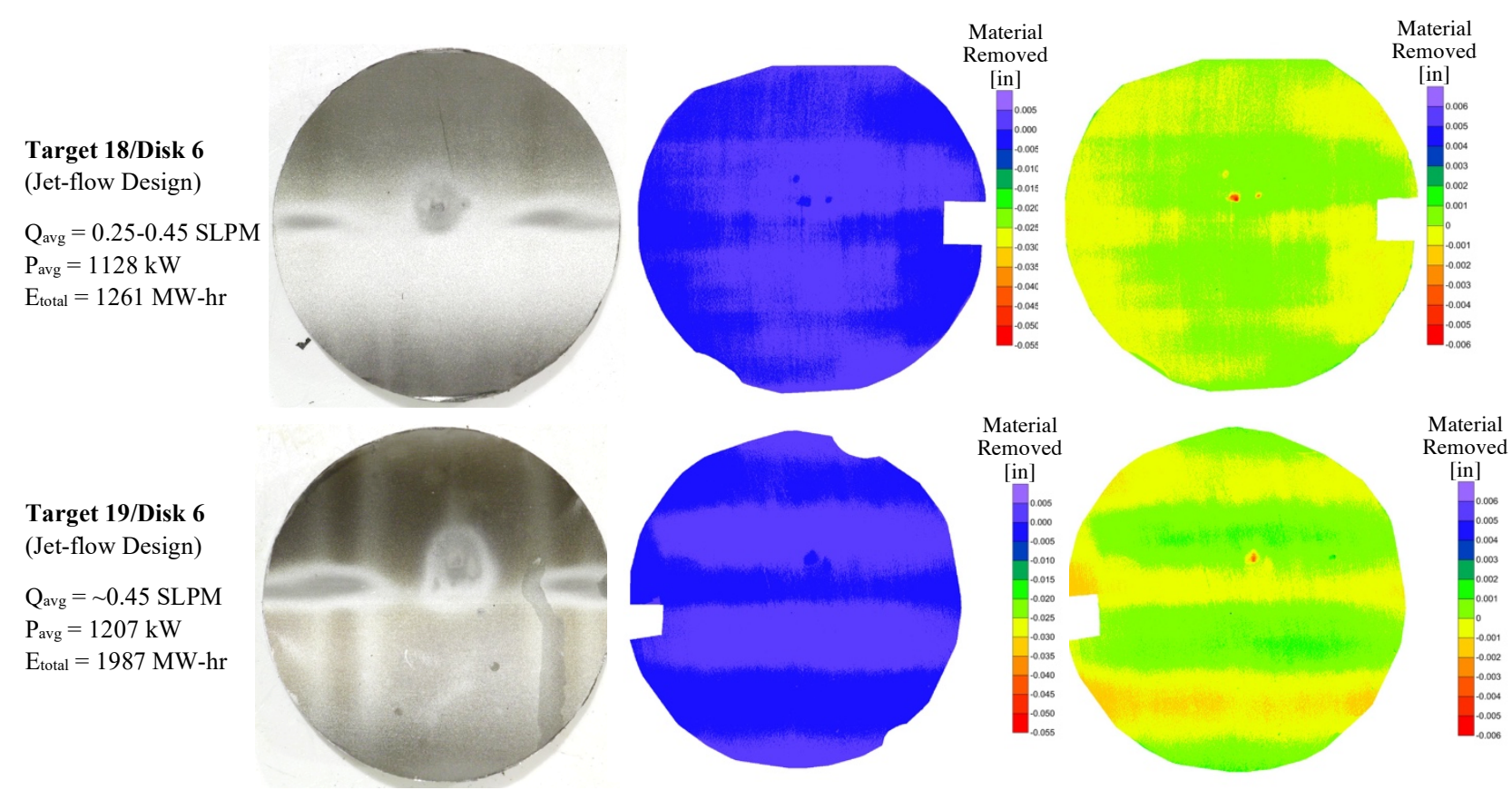

Figure 33. Images and erosion plots for Disk 6 samples from Targets 18 and 19 (jet-flow design with gas injection). 


\section{SUMMARY}

Disk-shaped samples removed from four different locations of SNS targets were scanned using a laser line scanning system to quantify the erosion damage that occurred during operation. The scan data were analyzed to calculate various erosion damage metrics for each sample. The results characterized and quantified the different erosion damage patterns observed in the two different target designs operated to date. The results and findings can be summarized as follows:

1. Erosion damage to the end of the mercury supply passages in original designed targets had two general types of erosion morphology: (1) relatively shallow ( $<0.010$ in) dimple-like pitting and (2) deep cavity-like erosion $(>0.050$ in).

2. "Comet-shaped" areas of discoloration were observed on the Disk 13/17 samples from the two jet-flow targets operated with gas-injection (Targets 18 and 19).

3. The location of maximum erosion were very similar for all Disk $13 / 17$ specimens scanned; all locations were within a 0.370 in diameter bounding circle when superimposed together on one figure.

4. Erosion damage to samples from targets operated with gas injection was less than the damage measured on targets operated without gas injection in all locations examined, quantifying the beneficial effect of gas injection on erosion damage.

\section{ACKNOWLEDGEMENTS}

The authors would like to thank Jill Hemman of Oak Ridge National Laboratory for her assistance with graphics included in this report, as well as Lawrence Crum (University of Washington) and FARO ${ }^{\circledR}$ Technologies Inc. for granting permission to use their images.

\section{REFERENCES}

[1] S.J. Pawel, J.R. DiStefano, E.T. Manneschmidt, Corrosion of type 316L stainless steel in a mercury thermal convection loop, Oak Ridge National Laboratory Technical Manuscript ORNL/TM-13754 (1999).

[2] J.D. Hunn, B.W. Riemer, C.C. Tsai, Journal of Nuclear Materials 318 (2003) 102-108. 
[3] K. Farrell, E.D. Specht, J. Pang, L.R. Walker, A. Rar, J.R. Mayotte, Journal of Nuclear Materials 343 (2005) 123-133.

[4] K. Farrell, T.S. Byun, Journal of Nuclear Materials 356 (2006) 178-188.

[5] David A. McClintock, James W. Hyres, Bradley J. Vevera, Journal of Nuclear Materials 450 (2014) 176-182.

[6] D.A. McClintock, P.D. Ferguson, L.K. Mansur, Journal of Nuclear Materials 398 (2010) 73-80.

[7] D.A. McClintock, B.W. Riemer, P.D. Ferguson, A.J. Carroll, M.J. Dayton, Journal of Nuclear Materials 431 (2012) 147-159.

[8] David A. McClintock, Bradley J. Vevera, Bernard W. Riemer, Franz X. Gallmeier, James W. Hyres, Phillip D. Ferguson, Journal of Nuclear Materials 450 (2014) 130-140.

[9] David McClintock, Observations of Cavitation-Induced Erosion of AISI 316L Target Vessels at the Spallation Neutron Source, SNS Technical Report 106010100-TR0001-R00, (October 2016).

[10] B. Riemer, J. Haines, M. Wendel, G. Bauer, M. Futakawa, S. Hasegawa, H. Kogawa, Journal of Nuclear Materials 377 (2008) 162-173.

[11] B. Riemer et al., Journal of Nuclear Materials 450 (2014) 192-203.

[12] J.R. Haines, B.W. Riemer, D.K. Felde, J.D. Hunn, S.J. Pawel, C.C. Tsai, Journal of Nuclear Materials 343 (2005) 58-69.

[13] Neutron Technologies Division, Target Management Plan, Spallation Neutron Source Technical Report 106010000-PN0005, R04 (July 2018).

[14] Wim Blokland, Yun. Liu, Mark Wendel, Drew Winder, Measurements of the Effects of Gas Injection into SNS Target 18, Spallation Neutron Source Technical Report 106010101-TR0043. 